\title{
An Analytic Approach for Pricing American Options with Regime Switching
}

\author{
Leunglung Chan ${ }^{1, *(1)}$ and Song-Ping Zhu ${ }^{2}$ \\ 1 School of Mathematics and Statistics, University of New South Wales, Sydney, NSW 2052, Australia \\ 2 School of Mathematics and Applied Statistics, University of Wollongong, Wollongong, NSW 2522, Australia; \\ spz@uow.edu.au \\ * Correspondence: leung.chan@unsw.edu.au
}

check for

updates

Citation: Chan, Leunglung, and Song-Ping Zhu. 2021. An Analytic Approach for Pricing American Options with Regime Switching. Journal of Risk and Financial Management 14: 188. https:// doi.org/10.3390/jrfm14050188

Academic Editor: Thanasis Stengos and Shigeyuki Hamori

Received: 25 January 2021

Accepted: 14 April 2021

Published: 21 April 2021

Publisher's Note: MDPI stays neutral with regard to jurisdictional claims in published maps and institutional affiliations.

Copyright: (c) 2021 by the authors. Licensee MDPI, Basel, Switzerland. This article is an open access article distributed under the terms and conditions of the Creative Commons Attribution (CC BY) license (https:/ / creativecommons.org/licenses/by/ $4.0 /)$.

\begin{abstract}
This paper investigates the American option price in a two-state regime-switching model. The dynamics of underlying are driven by a Markov-modulated Geometric Wiener process. That means the interest rate, the appreciation rate, and the volatility of underlying rely on hidden states of the economy which can be interpreted in terms of Markov chains. By means of the homotopy analysis method, an explicit formula for pricing two-state regime-switching American options is presented.
\end{abstract}

Keywords: option pricing; free boundary problem; regime switching; homotopy analysis method

\section{Introduction}

Pricing financial derivative is an ative field of research in mathematical finance. The pioneering work of Black and Scholes (1973) and Merton (1973) laid the foundations of field and gave rise tto a branch of the research in option pricing theory. The most options traded in the financial markets are American-style options. An American option grants its holder with the right to exercise the option before or on its expiry date. For pricing American options, one needs to determine the option price as well as its optimal exercise boundary. In the Black-Scholes framework, there is an analytical solution for pricing finite-horizon American options, which was first presented by Zhu (2006). Prior to the work of Zhu (2006), McKean (1965) and Merton (1973) pointed out that the problem of pricing American option is equivalent to a free-boundary problem. There are many methods, including analytical and numerical approximations, to solve this kind of free boundary problem. For instance, analytical approximation works include Johnson (1983); MacMillan (1986); Omberg (1987); Barone-Adesi and Whaley (1987); Barone-Adesi and Elliott (1991); Carr (1998) and Bunch and Johnson (2000). While numerical approximation work include Brennan and Schwartz (1977); Geske and Shastri (1985); Geske and Johnson (1984); Carr and Faguet (1996), and Hon and Mao (1997). An alternative approach aparting from a free boundary problem is called the decomposition method, see Jacka (1991); Kim (1990); Carr et al. (1992). Using the decomposition method, the price of an American option can be written as the sum of its European counterpart and the early exercise premium. Carr et al. (1992) proposed a variant decomposition approach, in which the price of American option can be written as its intrinsic value plus its time value. The early exercise premium is expressed in an integral form, and it implicitly relies on the early exercise boundary. The only known explicit solution for the optimal exercise boundary was found by Zhu (2006) in the form of infinite series. There are many approximation methods in the literature. In particular, the asymptotic behaviour of the optimal exercise boundary near maturity has attracted lots of attention as a promising way to derive an explicit formula, see Alobaidi and Mallier (2001); Chevalier (2005); Evans et al. (2002). However, these short-maturity asymptotic approximations of the optimal exercise boundary are not sufficiently precise under realistic model parameters, see Mallier (2002). The other numerical schemes for calculating the early exercise premium include Huang et al. (1996) and Ju (1998). 
Recently, there is active research in the applications of regime switching models followed by an unobservable Markov chain to a variety of financial problems. For an overview of unobservable Markov chains and their financial applications, we refer to Elliott et al. (1994) and Elliott and Kopp (1999). Some financial applications of regime switching include Elliott and van der Hoek (1997) for asset allocation, Elliott et al. (2001) for interest rate models, Buffington and Elliott (2002a, 2002b) for pricing European and American options, Elliott et al. (2003) for volatility estimation, Guo (2001a) for option pricing, Elliott et al. (2005) for option pricing via an Esscher transformation. In addition, Guo (2001b) investigated Russian options in the regime switching model. Guo and Shepp (2001) investigated the perpetual American lookback options. Guo and Zhang (2004) priced the perpetual American options with regime switching. Jobert and Rogers (2006) priced the finite maturity American put and barrier options with regime switching using a lattice approach. Huang (2011) formulated the regime-switching American problem as an optimal control problem and used discretization methods to solve it. Lu and Putri (2020) applied Laplace transform to regime-switch the American option and obtained a semianalytical solution. Egami and Kevkhishvili (2020) reduced an optimal stopping problem with an arbitrary value function in a two-regime environment to a pair of optimal stopping problems without regime switching. So far, there is no analytical formula for the finitehorizon regime switching American option.

Regime switching models have been used in finance practice and have recently started gaining increasing popularity. One of the key issues is how to identify the states of economy in regime switching models. In this regard, two recent publications have discussed this issue (see He and Zhu (2018) and He and Zhu (2021), in addition to using real market data in calibration, so that a practical use of regime switching models is clearly demonstrated through convincing empirical evidence. Another example that has clearly demonstrated the use of regime switching models in finance practice is the two earlier papers that were published by Elliott et al. (2003) and Elliott and Royal (2007), who used filtering methods to estimate the state of the economy from historical stock market data. In these two publications, a log-normal dynamics with Markov-switching volatility and a regime switching variance Gamma dynamics were adopted in their regime switching models, respectively.

We will extend the work of Zhu (2006) to obtain an explicit formula for pricing finite-horizon regime switching American options by means of the homotopy analysis method (HAM). HAM was first proposed by Ortega and Rheinboldt (1970) and has been successfully applied to solve a variety of heat transfer problems, see Liao (1997); Liao and Zhu (1999); and fluid-flow problems, see Liao and Zhu (1996); Liao and Campo (2002). Zhu (2006) applied HAM to derive an explicit pricing formula for American options in the Black-Scholes-Merton framework. Gounden and O'Hara (2010) extended the work of Zhu to pricing an American-style Asian option of floating strike type in the Black-ScholesMerton model. Leung (2013) applied HAM to obtain an explicit formula for lookback options under a stochastic volatility model. Chan and Zhu (2015) used HAM to derive an explicit formula for American-style convertible bonds with regime switching.

In this paper, we derive an explicit formula for pricing American options with regime switching. The paper is organized, as follows. Section 2 describes the model and formulates the problem. Section 3 presents the pricing of American options based on the HAM approach. The final section concludes.

\section{The Model Formulation}

Let $(\Omega, \mathcal{F}, \mathcal{P})$ be a complete probability space, where $\mathcal{P}$ is an actual probability measure. Let $\mathcal{T}$ be the time index set $[0, T]$ of the model. Let $\left\{W_{t}\right\}_{t \in \mathcal{T}}$ be a standard Wiener process on $(\Omega, \mathcal{F}, \mathcal{P})$. We consider a continuous-time Markov chain $\left\{X_{t}\right\}_{t \in \mathcal{T}}$ on $(\Omega, \mathcal{F}, \mathcal{P})$ with a finite state space $\mathcal{X}:=\left(x_{1}, x_{2}, \ldots, x_{N}\right)$. Without a loss of generality, we can identify the state space of $\left\{X_{t}\right\}_{t \in \mathcal{T}}$ to be a finite set of unit vectors $\left\{e_{1}, e_{2}, \ldots, e_{N}\right\}$, where $e_{i}=(0, \ldots, 1, \ldots, 0) \in \mathcal{R}^{\mathcal{N}}$. We assume that $\left\{X_{t}\right\}_{t \in \mathcal{T}}$ and $\left\{W_{t}\right\}_{t \in \mathcal{T}}$ are independent. 
Denote $A$ for the generator $\left[a_{i j}\right]_{i, j=1,2, \ldots, N}$. Subsequently, according to Elliott et al. (1994), the semi-martingale representation theorem is in the form of $\left\{X_{t}\right\}_{t \in \mathcal{T}}$ :

$$
X_{t}=X_{0}+\int_{0}^{t} A X_{s} d s+M_{t}
$$

Here, $\left\{M_{t}\right\}_{t \in \mathcal{T}}$ is an $\mathcal{R}^{N}$-valued martingale increment process with respect to the filtration that is generated by $\left\{X_{t}\right\}_{t \in \mathcal{T}}$.

The interest rate $\left\{r\left(t, X_{t}\right)\right\}_{t \in \mathcal{T}}$ of the money market account is given by:

$$
r_{t}:=r\left(t, X_{t}\right)=<r, X_{t}>,
$$

where $r:=\left(r_{1}, r_{2}, \ldots, r_{N}\right)$ with $r_{i}>0$ for each $i=1,2, \ldots, N$ and $<\cdot, \cdot>$ denotes the inner product in $\mathcal{R}^{N}$.

In this case, the dynamics of underlying $\left\{B_{t}\right\}_{t \in \mathcal{T}}$ for the money market account are given by:

$$
d B_{t}=r_{t} B_{t} d t, \quad B_{0}=1
$$

We suppose that the stock appreciation rate $\left\{\mu_{t}\right\}_{t \in \mathcal{T}}$ and the volatility $\left\{\sigma_{t}\right\}_{t \in \mathcal{T}}$ of $S$ also rely on $\left\{X_{t}\right\}_{t \in \mathcal{T}}$ and they are given by:

$$
\mu_{t}:=\mu\left(t, X_{t}\right)=<\mu, X_{t}>, \quad \sigma_{t}:=\sigma\left(t, X_{t}\right)=<\sigma, X_{t}>.
$$

Here, $\mu:=\left(\mu_{1}, \mu_{2}, \ldots, \mu_{N}\right)$ and $\sigma:=\left(\sigma_{1}, \sigma_{2}, \ldots, \sigma_{N}\right)$ with $\sigma_{i}>0$ for each $i=$ $1,2, \ldots, N$.

The dynamics of underlying $\left\{S_{t}\right\}_{t \in \mathcal{T}}$ are then given by the following Markov-modulated geometric Wiener process:

$$
d S_{t}=\mu_{t} S_{t} d t+\sigma_{t} S_{t} d W_{t}, \quad S_{0}=s
$$

Here, $W:=\left\{W_{t}\right\}$ is a standard Wiener process on a complete probability space $(\Omega, \mathcal{F}, \mathcal{P})$ with respect to the filtration $\left\{\mathcal{F}_{t}^{W}\right\}$, which is the $\mathcal{P}$-augmentation of the natural filtration that is generated by $W$.

\section{Free Boundary Problem}

Let us consider an American put option with expiry $T$ and exercise price $K$. In this subsection, we consider a special case with the number of regimes $N$, being 2 in order to simplify our discussion. We adopt the approach in Buffington and Elliott (2002b) to derive the results in this section. Given that $S_{t}=S$ and that $X_{t}=X$, the price of the American put option at time $t$ is given by:

$$
V(t, T, S, X)=\sup _{\tau \in[t, T]} E_{\mathcal{Q}}\left[\exp \left(-\int_{t}^{T} r_{u} d u\right)(K-S)^{+} \mid S_{t}=S, X_{t}=X\right]
$$

where the supremum is taken over the set of stopping times $\tau$ taking values in $[t, T]$.

Let $\mathbf{V}:=\mathbf{V}(t, T, S)=\left(V\left(t, T, S, e_{1}\right), V\left(t, T, S, e_{2}\right)\right)=\left(V_{1}, V_{2}\right)$. When $X_{t}=e_{i}(i=1,2)$, the continuation region is given by:

$$
\mathcal{C}^{i}=\left\{(S, t) \in \mathcal{R}^{+} \times[0, T] \mid V\left(t, T, S, e_{i}\right)>(K-S)^{+}\right\}
$$

and the stopping region is given by:

$$
\mathcal{S}^{i}=\left\{(S, t) \in \mathcal{R}^{+} \times[0, T] \mid V\left(t, T, S, e_{i}\right)=(K-S)^{+}\right\} .
$$

Follow the lines of Buffington and Elliott (2002b), we write $\mathcal{C}_{t}^{i}$ for the $t$-section of $\mathcal{C}^{i}$, for each $i=1,2$ and $t \in[0, T]$. Let $S_{f_{i}}(t)=S_{f}\left(t, e_{i}\right)$ be the critical price of the American 
put when $X_{t}=e_{i}(i=1,2)$; that is, when the state $X_{t}=e_{i}$ and the price of the underlying at time $t$ falls below $S_{f_{i}}(t)$, it is rational for the holder of the American put to exercise the option at time $t$. Note that $S_{f_{i}}(T)=K$, for each $i=1,2$. Subsequently, by Elliott and Kopp (1999) and Buffington and Elliott (2002b),

$$
\mathcal{C}_{t}^{i}=\left(S_{f_{i}}(t), \infty\right), \quad i=1,2 .
$$

We shall consider the case $N=2$.

Yi (2008) showed that, if $\sigma_{1}>\sigma_{2}$ then $V_{1} \geq V_{2}$ and $S_{f_{1}}(t) \leq S_{f_{2}}(t)$ for each $t \in[0, T]$ in a two-state regime switching model. When $S>S_{f_{2}}(t),(S, t)$ is in the common continuation region $\mathcal{C}^{1} \cup \mathcal{C}^{2}$ and $\mathbf{V}=\left(V_{1}, V_{2}\right)$ satisfies the following pair of coupled P.D.E.s:

$$
\begin{aligned}
& \frac{\partial V_{1}}{\partial t}+r_{1} S \frac{\partial V_{1}}{\partial S}+\frac{1}{2} \sigma_{1}^{2} S^{2} \frac{\partial^{2} V_{1}}{\partial S^{2}}-r_{1} V_{1}+<\mathbf{V}, A e_{1}>=0, \\
& \frac{\partial V_{2}}{\partial t}+r_{2} S \frac{\partial V_{2}}{\partial S}+\frac{1}{2} \sigma_{2}^{2} S^{2} \frac{\partial^{2} V_{2}}{\partial S^{2}}-r_{2} V_{2}+<\mathbf{V}, A e_{2}>=0 .
\end{aligned}
$$

When $S \leq S_{f_{1}}(t)$,

$$
V_{1}=V\left(t, T, S, e_{1}\right)=K-S .
$$

When $S \leq S_{f_{2}}(t)$,

$$
V_{2}=V\left(t, T, S, e_{2}\right)=K-S .
$$

On the optimal curve $S_{f_{i}}(t)(i=1,2)$, it is required that $V_{i}$ satisfies a high-contact condition:

$$
V_{i}\left(t, T, S_{f_{i}}(t)\right)=K-S_{f_{i}}(t),
$$

and a smooth-pasting condition:

$$
\frac{\partial V_{i}}{\partial S}\left(t, T, S_{f_{i}}(t)\right)=-1 .
$$

When $S$ is in the transition region $\left\{(S, t) \mid S_{f_{1}}(t) \leq S \leq S_{f_{2}}(t)\right\}, V_{2}=K-S$ and $V_{1}$ satisfies the following P.D.E.:

$$
\frac{\partial V_{1}}{\partial t}+r_{1} S \frac{\partial V_{1}}{\partial S}+\frac{1}{2} \sigma_{1}^{2} S^{2} \frac{\partial^{2} V_{1}}{\partial S^{2}}-r_{1} V_{1}+a_{11} V_{1}-a_{11}(K-S)=0,
$$

and boundary conditions

$$
\begin{gathered}
V_{1}\left(t, T, S_{f_{1}}(t)\right)=K-S_{f_{1}}(t) \\
\frac{\partial V_{1}}{\partial S}\left(t, T, S_{f_{1}}(t)\right)=-1 . \\
V_{1}(T, T, S)=V\left(T, T, S, e_{1}\right)=\max \{K-S, 0\} .
\end{gathered}
$$

Clearly, there are two free boundary functions, $S_{f_{1}}(t)$ and $S_{f_{2}}(t)$, and two option prices, $V_{1}(S, t)$ and $V_{2}(S, t)$, as listed in the above PDE systems. Which one of these is used to determine if an option should be exercised or continuously held has not been clearly stated in the literature. A flow chart could be useful for financial practitioners who wish to use our solutions to price American put options in a two-state regime switching setting. 
Assumming that all of the model parameters, including $\sigma_{1}$ and $\sigma_{2}$, have been extracted from market data and assumming that one knows the current status of economy, i.e., we are either in State 1 or State 2, before one decides to use a regime-switching model to price an American option. Subsequently, the actual pricing exercise needs be divided into two stages, relying on the current status of economy when a pricing task is conducted. If we are in a higher status of economy, i.e., in State 2, using the $S_{f_{2}}^{(1)}$ obtained in Section 3.1 would be sufficient, because, in this case, once $S$ drops below $S_{f_{2}}^{(1)}$ from the above, the put option should be exercised as a result of the fact that $S_{f_{1}}^{(1)}(t) \leq S_{f_{2}}^{(1)}(t)$. On the other hand, if we are in a lower status of economy, i.e., in State 1, an additional PDE system may need to be solved relying on the current underlying value. For the range of $S_{f_{1}}^{(1)}(t) \leq S \leq S_{f_{2}}^{(1)}(t)$, the solution that is shown in Section 3.1 can no longer be used and one needs to use the solution shown in Section 3.2, in which $V_{2}$ has already "landed" on the payoff function. This pricing procedure can be better demonstrated with the following flow chart, see Figure 1.

Note that we use superscripts in the flow chart to denote the free boundaries that were obtained in the corresponding stage. For simplicity, they will all be removed in the next section without any ambiguity, as we have denoted each stage by a separate subsection.

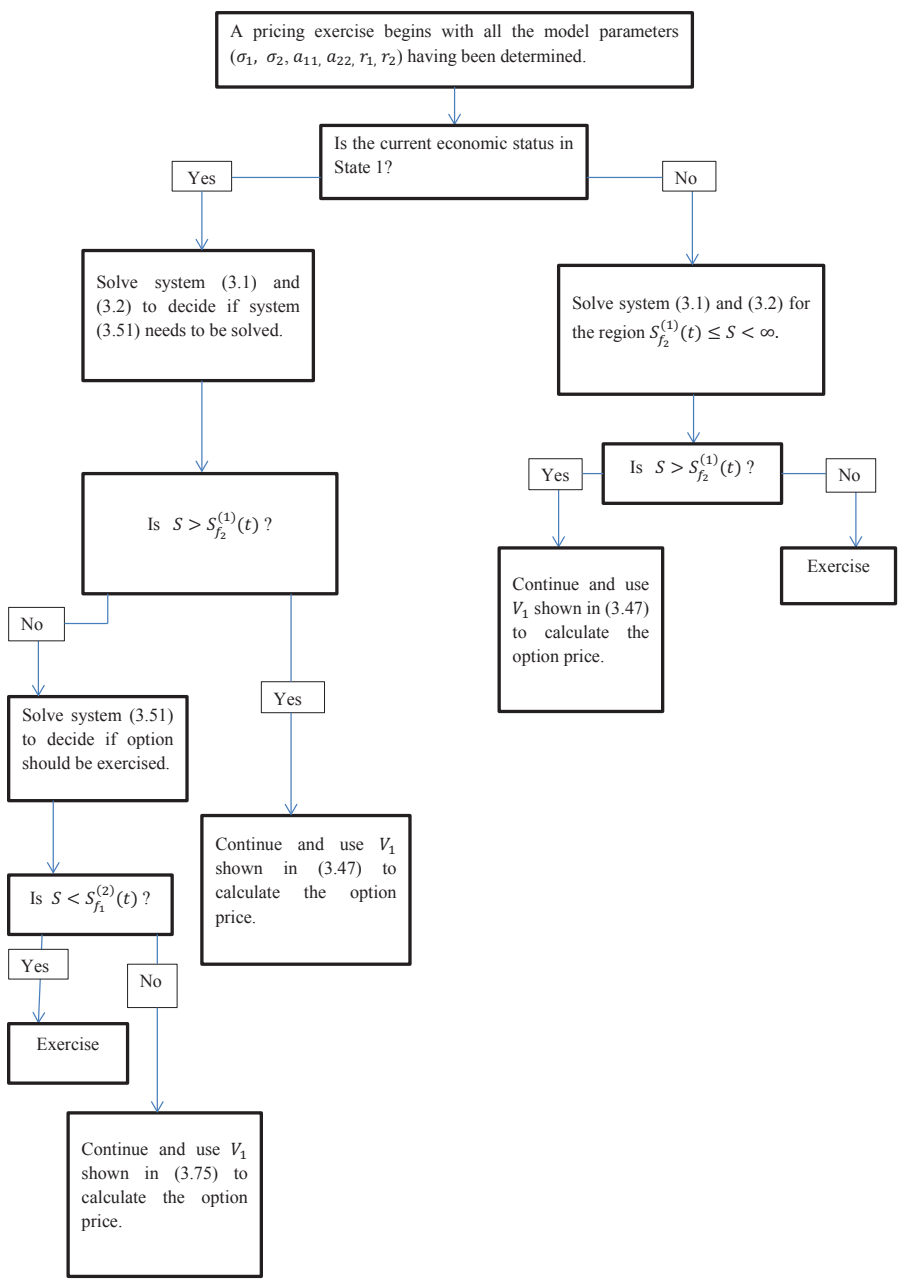

Figure 1. An illustrative flow chart for the usage of our solutions. 


\section{HAM Approach}

It was noted earlier that American options can be formulated as free boundary problems. Closed-form solutions of free boundary problems are notoriously difficult to derive. However, by employing the homotopy analysis method, an exact and closed-form solution can be derived, in the form of infinite series. We have defined the common continuation region and the transition region in Section 2. Each problem in these regions is solved below.

\subsection{The Common Continuation Region}

Firstly, let us discuss the American put value function in the common continuation region where $S>S_{f_{2}}(t)$ and $V_{1}, V_{2}$ satisfy the equations:

$$
\begin{aligned}
& \left\{\begin{array}{l}
\frac{\partial V_{1}}{\partial t}+r_{1} S \frac{\partial V_{1}}{\partial S}+\frac{1}{2} \sigma_{1}^{2} S^{2} \frac{\partial^{2} V_{1}}{\partial S^{2}}-r_{1} V_{1}+<\mathbf{V}, A e_{1}>=0 \\
V_{1}\left(t, T, S_{f_{1}}(t)\right)=K-S_{f_{1}}(t) \\
\frac{\partial V_{1}}{\partial S}\left(t, T, S_{f_{1}}(t)\right)=-1 \\
V_{1}(T, T, S)=V\left(T, T, S, e_{1}\right)=\max \{K-S, 0\} \\
\lim _{S \rightarrow \infty} V_{1}(t, T, S)=0
\end{array}\right. \\
& \left\{\begin{array}{l}
\frac{\partial V_{2}}{\partial t}+r_{2} S \frac{\partial V_{2}}{\partial S}+\frac{1}{2} \sigma_{2}^{2} S^{2} \frac{\partial^{2} V_{2}}{\partial S^{2}}-r_{2} V_{2}+<\mathbf{V}, A e_{2}>=0 \\
V_{2}\left(t, T, S_{f_{2}}(t)\right)=K-S_{f_{2}}(t) \\
\frac{\partial V_{2}}{\partial S}\left(t, T, S_{f_{2}}(t)\right)=-1 \\
V_{2}(T, T, S)=V\left(T, T, S, e_{2}\right)=\max \{K-S, 0\} \\
\lim _{S \rightarrow \infty} V_{2}(t, T, S)=0
\end{array}\right.
\end{aligned}
$$

Note that $V_{i}$ and $S_{f_{i}}$ are coupled. To solve these systems effectively, we shall make all variables dimensionless by introducing dimensionless variables

$$
V_{i}^{\prime}=\frac{V_{i}}{K}, \quad S^{\prime}=\frac{S}{K}, \quad \tau_{i}^{\prime}=\frac{\sigma_{i}^{2}}{2}(T-t) \quad \text { for } \quad i=1,2 .
$$

With all of the primes dropped from now on, the dimensionless systems read

$$
\begin{gathered}
\left\{\begin{array}{l}
-\frac{\partial V_{1}}{\partial \tau_{1}}+\gamma_{1} S \frac{\partial V_{1}}{\partial S}+S^{2} \frac{\partial^{2} V_{1}}{\partial S^{2}}-\gamma_{1} V_{1}=\lambda_{1}\left(V_{2}-V_{1}\right) \\
V_{1}\left(\tau_{1}, S_{f_{1}}\left(\tau_{1}\right)\right)=1-S_{f_{1}}\left(\tau_{1}\right) \\
\frac{\partial V_{1}}{\partial S}\left(\tau_{1}, S_{f_{1}}\left(\tau_{1}\right)\right)=-1 \\
V_{1}(0, S)=V\left(0, S, e_{1}\right)=\max \{1-S, 0\} \\
\lim _{S \rightarrow \infty} V_{1}\left(\tau_{1}, S\right)=0
\end{array}\right. \\
\left\{\begin{array}{l}
-\frac{\partial V_{2}}{\partial \tau_{2}}+\gamma_{2} S \frac{\partial V_{2}}{\partial S}+S^{2} \frac{\partial^{2} V_{2}}{\partial S^{2}}-\gamma_{2} V_{2}=\lambda_{2}\left(V_{1}-V_{2}\right) \\
V_{2}\left(\tau_{2}, S_{f_{2}}\left(\tau_{2}\right)\right)=1-S_{f_{2}}\left(\tau_{2}\right) \\
\frac{\partial V_{2}}{\partial S}\left(\tau_{2}, S_{f_{2}}\left(\tau_{2}\right)\right)=-1 \\
V_{2}(0, S)=V\left(0, S, e_{2}\right)=\max \{1-S, 0\} \\
\lim _{S \rightarrow \infty} V_{2}\left(\tau_{2}, S\right)=0
\end{array}\right.
\end{gathered}
$$

Here, $\lambda_{i}=\frac{2 a_{i i}}{\sigma_{i}^{2}}, i=1,2$ and $\gamma_{i}=\frac{2 r_{i}}{\sigma_{i}^{2}}, i=1,2$, which can be viewed as an interest rate relative to the volatility of the underlying asset price.

Take the Laudau transformation

$$
x_{i}=\ln \left(\frac{S}{S_{f_{i}}\left(\tau_{i}\right)}\right)
$$


to change the moving boundary to fixed boundary conditions, thus yielding:

$$
\begin{aligned}
& \left\{\begin{array}{l}
\frac{\partial V_{1}}{\partial \tau_{1}}-\left(\gamma_{1}-1\right) \frac{\partial V_{1}}{\partial x_{1}}-\frac{\partial^{2} V_{1}}{\partial x_{1}^{2}}+\gamma_{1} V_{1}=\lambda_{1}\left(V_{1}-V_{2}\right)+\frac{1}{S_{f_{1}}\left(\tau_{1}\right)} \frac{d S_{f_{1}}\left(\tau_{1}\right)}{d \tau_{1}} \frac{\partial V_{1}}{\partial x_{1}} \\
\frac{\partial V_{1}}{\partial x_{1}}\left(\tau_{1}, 0\right)=-S_{f_{1}}\left(\tau_{1}\right) \\
V_{1}\left(0, x_{1}\right)=V\left(0, x_{1}, e_{1}\right)=0 \\
\lim _{x_{1} \rightarrow \infty} V_{1}\left(\tau_{1}, x_{1}\right)=0
\end{array}\right. \\
& \left\{\begin{array}{l}
\frac{\partial V_{2}}{\partial \tau_{2}}-\left(\gamma_{2}-1\right) \frac{\partial V_{2}}{\partial x_{2}}-\frac{\partial^{2} V_{2}}{\partial x_{2}^{2}}+\gamma_{2} V_{2}=\lambda_{2}\left(V_{2}-V_{1}\right)+\frac{1}{S_{f_{2}}\left(\tau_{2}\right)} \frac{d S_{f_{2}}\left(\tau_{2}\right)}{d \tau_{2}} \frac{\partial V_{2}}{\partial x_{2}} \\
V_{2}\left(\tau_{2}, 0\right)=1-S_{f_{2}}\left(\tau_{2}\right) \\
\frac{\partial V_{2}}{\partial x_{2}}\left(\tau_{2}, 0\right)=-S_{f_{2}}\left(\tau_{2}\right) \\
V_{2}\left(0, x_{2}\right)=V\left(0, x_{2}, e_{2}\right)=0 \\
\lim _{x_{2} \rightarrow \infty} V_{2}\left(\tau_{2}, x_{2}\right)=0
\end{array}\right.
\end{aligned}
$$

Now, to solve (23) and (24) by the homotopy analysis method, we introduce an embedding parameter $p \in[0,1]$ and construct unknown functions $\bar{V}_{i}\left(\tau_{i}, x_{i}, p\right)$ and $\bar{S}_{f_{i}}\left(\tau_{i}, p\right), i=$ 1,2 that satisfy the following differential systems:

$$
\begin{aligned}
& \left\{\begin{array}{l}
(1-p) \mathcal{L}_{1}\left[\bar{V}_{1}\left(\tau_{1}, x_{1}, p\right)-\bar{V}_{1}^{0}\left(\tau_{1}, x_{1}\right)\right]=-p\left\{\mathcal{A}_{1}\left[\bar{V}_{1}\left(\tau_{1}, x_{1}, p\right), \bar{S}_{f_{1}}\left(\tau_{1}, p\right)\right]\right\} \\
\bar{V}_{1}\left(0, x_{1}, p\right)=(1-p) \bar{V}_{1}^{0}\left(0, x_{1}\right) \\
\bar{V}_{1}\left(\tau_{1}, 0, p\right)+\bar{S}_{f_{1}}\left(\tau_{1}, p\right)=1 \\
\frac{\partial \bar{V}_{1}}{\partial x_{1}}\left(\tau_{1}, 0, p\right)+\bar{S}_{f_{1}}\left(\tau_{1}, p\right)=(1-p)\left[1+\frac{\partial \bar{V}_{1}^{0}}{\partial x_{1}}\left(\tau_{1}, 0\right)-\bar{V}_{1}^{0}\left(\tau_{1}, 0\right)\right] \\
\lim _{x_{1} \rightarrow \infty} \bar{V}_{1}\left(\tau_{1}, x_{1}, p\right)=0
\end{array}\right. \\
& \left\{\begin{array}{l}
(1-p) \mathcal{L}_{2}\left[\bar{V}_{2}\left(\tau_{2}, x_{2}, p\right)-\bar{V}_{2}^{0}\left(\tau_{2}, x_{2}\right)\right]=-p\left\{\mathcal{A}_{2}\left[\bar{V}_{2}\left(\tau_{2}, x_{2}, p\right), \bar{S}_{f_{2}}\left(\tau_{2}, p\right)\right]\right\} \\
\bar{V}_{2}\left(0, x_{2}, p\right)=(1-p) \bar{V}_{2}^{0}\left(0, x_{2}\right) \\
\bar{V}_{2}\left(\tau_{2}, 0, p\right)+\bar{S}_{f_{2}}\left(\tau_{2}, p\right)=1 \\
\frac{\partial \bar{V}_{2}}{\partial x_{2}}\left(\tau_{2}, 0, p\right)+\bar{S}_{f_{2}}\left(\tau_{2}, p\right)=(1-p)\left[1+\frac{\partial \bar{V}_{2}^{0}}{\partial x_{2}}\left(\tau_{2}, 0\right)-\bar{V}_{2}^{0}\left(\tau_{2}, 0\right)\right] \\
\lim _{x_{2} \rightarrow \infty} \bar{V}_{2}\left(\tau_{2}, x_{2}, p\right)=0
\end{array}\right.
\end{aligned}
$$

Here, $\mathcal{L}_{i}$ is a differential operator that is defined as

$$
\mathcal{L}_{i}=\frac{\partial}{\partial \tau_{i}}-\left(\gamma_{i}-1\right) \frac{\partial}{\partial x_{i}}-\frac{\partial^{2}}{\partial x_{i}^{2}}+\gamma_{i}
$$

and $\mathcal{A}_{i}$ is a functional defined as

$$
\begin{aligned}
& \mathcal{A}_{1}\left[\bar{V}_{1}\left(\tau_{1}, x_{1}, p\right), \bar{S}_{f_{1}}\left(\tau_{1}, p\right)\right] \\
= & \mathcal{L}_{1}\left(\bar{V}_{1}\right)-\lambda_{1}\left(\bar{V}_{1}-\bar{V}_{2}\right)-\frac{1}{\bar{S}_{f_{1}}\left(\tau_{1}, p\right)} \frac{d \bar{S}_{f_{1}}\left(\tau_{1}, p\right)}{d \tau_{1}} \frac{\partial \bar{V}_{1}}{\partial x_{1}}\left(\tau_{1}, x_{1}, p\right) \\
& \mathcal{A}_{2}\left[\bar{V}_{2}\left(\tau_{2}, x_{2}, p\right), \bar{S}_{f_{2}}\left(\tau_{2}, p\right)\right] \\
= & \mathcal{L}_{2}\left(\bar{V}_{2}\right)-\lambda_{2}\left(\bar{V}_{2}-\bar{V}_{1}\right)-\frac{1}{\bar{S}_{f_{2}}\left(\tau_{2}, p\right)} \frac{d \bar{S}_{f_{2}}\left(\tau_{2}, p\right)}{d \tau_{2}} \frac{\partial \bar{V}_{2}}{\partial x_{2}}\left(\tau_{2}, x_{2}, p\right)
\end{aligned}
$$

Equations (25) and (26) are known as the zeroth order deformation equations and they determine the continuous deformation of $\bar{V}_{i}\left(\tau_{i}, x_{i}, p\right)$. To construct Equations (25) and (26), 
we want to find a continuous map, such that the solution we are seeking becomes the result of a continuous deformation from an initial and known function. Here, the initial state of the continuous deformation is at $p=0$. With $p=0$, we have, from Equations (25) and (26),

$$
\begin{aligned}
& \left\{\begin{array}{l}
\mathcal{L}_{1}\left[\bar{V}_{1}\left(\tau_{1}, x_{1}, 0\right)\right]=\mathcal{L}_{1}\left[\bar{V}_{1}^{0}\left(\tau_{1}, x_{1}\right)\right] \\
\bar{V}_{1}\left(0, x_{1}, 0\right)=\bar{V}_{1}^{0}\left(0, x_{1}\right) \\
\bar{V}_{1}\left(\tau_{1}, 0,0\right)+\bar{S}_{f_{1}}\left(\tau_{1}, 0\right)=1 \\
\frac{\partial \bar{V}_{1}}{\partial x_{1}}\left(\tau_{1}, 0,0\right)+\bar{S}_{f_{1}}\left(\tau_{1}, 0\right)=\left[1+\frac{\partial \bar{V}_{1}^{0}}{\partial x_{1}}\left(\tau_{1}, 0\right)-\bar{V}_{1}^{0}\left(\tau_{1}, 0\right)\right] \\
\lim _{x_{1} \rightarrow \infty} \bar{V}_{1}\left(\tau_{1}, x_{1}, 0\right)=0
\end{array}\right. \\
& \left\{\begin{array}{l}
\mathcal{L}_{2}\left[\bar{V}_{2}\left(\tau_{2}, x_{2}, 0\right)\right]=\mathcal{L}_{2}\left[\bar{V}_{2}^{0}\left(\tau_{2}, x_{2}\right)\right] \\
\bar{V}_{2}\left(0, x_{2}, 0\right)=\bar{V}_{2}^{0}\left(0, x_{2}\right) \\
\bar{V}_{2}\left(\tau_{2}, 0,0\right)+\bar{S}_{f_{2}}\left(\tau_{2}, 0\right)=1 \\
\frac{\partial \bar{V}_{2}}{\partial x_{2}}\left(\tau_{2}, 0,0\right)+\bar{S}_{f_{2}}\left(\tau_{2}, 0\right)=\left[1+\frac{\partial \bar{V}_{2}^{0}}{\partial x_{2}}\left(\tau_{2}, 0\right)-\bar{V}_{2}^{0}\left(\tau_{2}, 0\right)\right] \\
\lim _{x_{2} \rightarrow \infty} \bar{V}_{2}\left(\tau_{2}, x_{2}, 0\right)=0
\end{array}\right.
\end{aligned}
$$

Clearly, the solutions of the differential systems (30) and (31) are in forms of

$$
\begin{aligned}
& \left\{\begin{array}{l}
\bar{V}_{1}\left(\tau_{1}, x_{1}, 0\right)=\bar{V}_{1}^{0}\left(\tau_{1}, x_{1}\right) \\
\bar{S}_{f_{1}}\left(\tau_{1}, 0\right)=1-\bar{V}_{1}^{0}\left(\tau_{1}, 0\right)=\bar{S}^{0}\left(\tau_{1}\right)
\end{array}\right. \\
& \left\{\begin{array}{l}
\bar{V}_{2}\left(\tau_{2}, x_{2}, 0\right)=\bar{V}_{2}^{0}\left(\tau_{2}, x_{2}\right) \\
\bar{S}_{f_{2}}\left(\tau_{2}, 0\right)=1-\bar{V}_{2}^{0}\left(\tau_{2}, 0\right)=\bar{S}^{0}\left(\tau_{2}\right)
\end{array}\right.
\end{aligned}
$$

so long as the initial guess $\bar{V}_{i}^{0}\left(\tau_{i}, x_{i}\right)$ satisfies the condition

$$
\lim _{x_{i} \rightarrow \infty} \bar{V}_{i}^{0}\left(\tau_{i}, x_{i}\right)=0 .
$$

Note that, other than this condition, theoretically, there are no other requirements for the initial guess $\bar{V}_{i}^{0}\left(\tau_{i}, x_{i}\right)$ to satisfy. However, a faster convergence of the series is expected if we select a function that has already satisfied an additional condition $\mathcal{L}_{i}\left[\bar{V}^{0}\left(\tau_{i}, x_{i}\right)\right]=0$.

With $p=1$, we have

$$
\begin{aligned}
& \left\{\begin{array}{l}
\mathcal{L}_{1}\left[\bar{V}_{1}\left(\tau_{1}, x_{1}, 1\right)\right]=\lambda_{1}\left(\bar{V}_{1}\left(\tau_{1}, x_{1}, 1\right)-\bar{V}_{2}\left(\tau_{2}, x_{2}, 1\right)\right)+\frac{1}{\bar{S}_{f_{1}}\left(\tau_{1}, 1\right)} \frac{d \bar{S}_{f_{1}}\left(\tau_{1}, 1\right)}{d \tau_{1}} \frac{\partial \bar{V}_{1}}{\partial x_{1}}\left(\tau_{1}, x_{1}, 1\right) \\
\bar{V}_{1}\left(0, x_{1}, 1\right)=0 \\
\bar{V}_{1}\left(\tau_{1}, 0,1\right)=1-\bar{S}_{f_{1}}\left(\tau_{1}, 1\right) \\
\frac{\partial \bar{V}_{1}}{\partial x_{1}}\left(\tau_{1}, 0,1\right)=-\bar{S}_{f_{1}}\left(\tau_{1}, 1\right) \\
\lim _{x_{1} \rightarrow \infty} \bar{V}_{1}\left(\tau_{1}, x_{1}, 1\right)=0
\end{array}\right. \\
& \left\{\begin{array}{l}
\mathcal{L}_{2}\left[\bar{V}_{2}\left(\tau_{2}, x_{2}, 1\right)\right]=\lambda_{2}\left(\bar{V}_{2}\left(\tau_{2}, x_{2}, 1\right)-\bar{V}_{1}\left(\tau_{1}, x_{1}, 1\right)\right)+\frac{1}{\bar{S}_{f_{2}}\left(\tau_{2}, 1\right)} \frac{d \bar{S}_{f_{2}}\left(\tau_{2}, 1\right)}{d \tau_{2}} \frac{\partial \bar{V}_{2}}{\partial x_{2}}\left(\tau_{2}, x_{2}, 1\right) \\
\bar{V}_{2}\left(0, x_{2}, 1\right)=0 \\
\bar{V}_{2}\left(\tau_{2}, 0,1\right)=1-\bar{S}_{f_{2}}\left(\tau_{2}, 1\right) \\
\frac{\partial \bar{V}_{2}}{\partial x_{2}}\left(\tau_{2}, 0,1\right)=-\bar{S}_{f_{2}}\left(\tau_{2}, 1\right) \\
\lim _{x_{2} \rightarrow \infty} \bar{V}_{2}\left(\tau_{2}, x_{2}, 1\right)=0
\end{array}\right.
\end{aligned}
$$

Comparing with (23) and (24), it is easy to see that the solution we seek is nothing but

$$
\left\{\begin{array}{l}
V_{1}\left(\tau_{1}, x_{1}\right)=\bar{V}_{1}\left(\tau_{1}, x_{1}, 1\right) \\
S_{f_{1}}\left(\tau_{1}\right)=\bar{S}_{f_{1}}\left(\tau_{1}, 1\right)
\end{array}\right.
$$




$$
\left\{\begin{array}{l}
V_{2}\left(\tau_{2}, x_{2}\right)=\bar{V}_{2}\left(\tau_{2}, x_{2}, 1\right) \\
S_{f_{2}}\left(\tau_{2}\right)=\bar{S}_{f_{2}}\left(\tau_{2}, 1\right)
\end{array}\right.
$$

In order to determine the values of $\bar{V}_{i}\left(\tau_{i}, x_{i}, 1\right)$ and $\bar{S}_{f_{i}}\left(\tau_{i}, 1\right), i=1,2$, we can apply a Taylor's series expansion of $p$ to the functions $\bar{V}_{i}\left(\tau_{i}, x_{i}, p\right)$ and $\bar{S}_{f_{i}}\left(\tau_{i}, p\right)$

$$
\begin{gathered}
\left\{\begin{array}{l}
\bar{V}_{1}\left(\tau_{1}, x_{1}, p\right)=\sum_{n=0}^{\infty} \frac{\bar{V}_{1}^{n}\left(\tau_{1}, x_{1}\right)}{n !} p^{n} \\
\bar{S}_{f_{1}}\left(\tau_{1}, p\right)=\sum_{n=0}^{\infty} \frac{\overline{\bar{S}}^{n}\left(\tau_{1}\right)}{n !} p^{n}
\end{array}\right. \\
\left\{\begin{array}{l}
\bar{V}_{2}\left(\tau_{2}, x_{2}, p\right)=\sum_{n=0}^{\infty} \frac{\bar{V}_{2}^{n}\left(\tau_{2}, x_{2}\right)}{n !} p^{n} \\
\bar{S}_{f_{2}}\left(\tau_{2}, p\right)=\sum_{n=0}^{\infty} \frac{\bar{S}^{n}\left(\tau_{2}\right)}{n !} p^{n}
\end{array}\right.
\end{gathered}
$$

where

$$
\begin{aligned}
& \left\{\begin{array}{l}
\bar{V}_{1}^{n}\left(\tau_{1}, x_{1}\right)=\left.\frac{\partial^{n}}{\partial p^{n}} \bar{V}_{1}\left(\tau_{1}, x_{1}, p\right)\right|_{p=0} \\
\bar{S}^{n}\left(\tau_{1}\right)=\left.\frac{\partial^{n}}{\partial p^{n}} \bar{S}_{f_{1}}\left(\tau_{1}, p\right)\right|_{p=0}
\end{array}\right. \\
& \left\{\begin{array}{l}
\bar{V}_{2}^{n}\left(\tau_{2}, x_{2}\right)=\left.\frac{\partial^{n}}{\partial p^{n}} \bar{V}_{2}\left(\tau_{2}, x_{2}, p\right)\right|_{p=0} \\
\bar{S}^{n}\left(\tau_{2}\right)=\left.\frac{\partial^{n}}{\partial p^{n}} \bar{S}_{f_{2}}\left(\tau_{2}, p\right)\right|_{p=0}
\end{array}\right.
\end{aligned}
$$

To determine the coefficients in the above Taylor series expansion, we derive a set of governing equations by differentiating the zeroth-order (25) and (26), with respect to $p$ and then set $p=0$. After this process, we get

$$
\left\{\begin{array}{l}
\mathcal{L}_{1}\left[\bar{V}_{1}^{1}\left(\tau_{1}, x_{1}\right)\right]=-\mathcal{L}_{1}\left[\bar{V}_{1}^{0}\left(\tau_{1}, x_{1}\right)\right]+\tilde{\mathcal{A}}_{1}\left(\tau_{1}, x_{1}, 0\right) \\
\bar{V}_{1}^{1}\left(0, x_{1}\right)=-\bar{V}_{1}^{0}\left(0, x_{1}\right) \\
\bar{V}_{1}^{1}\left(\tau_{1}, 0\right)+\bar{S}^{1}\left(\tau_{1}\right)=0 \\
\frac{\partial V_{1}^{1}}{\partial x_{1}}\left(\tau_{1}, 0\right)+\bar{S}^{1}\left(\tau_{1}\right)=\bar{V}_{1}^{0}\left(\tau_{1}, 0\right)-\frac{\partial \bar{V}_{1}^{0}}{\partial x_{1}}\left(\tau_{1}, 0\right)-1 \\
\lim _{x_{1} \rightarrow \infty} \bar{V}_{1}^{1}\left(\tau_{1}, x_{1}\right)=0
\end{array}\right.
$$

and

$$
\left\{\begin{array}{l}
\mathcal{L}_{1}\left[\bar{V}_{1}^{n}\left(\tau_{1}, x_{1}\right)\right]=\left.n \frac{\partial^{n-1} \tilde{\mathcal{A}}_{1}}{\partial p^{n-1}}\right|_{p=0} \\
\bar{V}_{1}^{n}\left(0, x_{1}\right)=0 \\
\bar{V}_{1}^{n}\left(\tau_{1}, 0\right)+\bar{S}^{n}\left(\tau_{1}\right)=0, \quad \text { if } \quad n \geq 2 \\
\frac{\partial \bar{V}_{1}^{n}}{\partial x_{1}}\left(\tau_{1}, 0\right)+\bar{S}^{n}\left(\tau_{1}\right)=0 \\
\lim _{x_{1} \rightarrow \infty} \bar{V}_{1}^{n}\left(\tau_{1}, x_{1}\right)=0
\end{array}\right.
$$

Additionally,

$$
\left\{\begin{array}{l}
\mathcal{L}_{2}\left[\bar{V}_{2}^{1}\left(\tau_{2}, x_{2}\right)\right]=-\mathcal{L}_{2}\left[\bar{V}_{2}^{0}\left(\tau_{2}, x_{2}\right)\right]+\tilde{\mathcal{A}}_{2}\left(\tau_{2}, x_{2}, 0\right) \\
\bar{V}_{2}^{1}\left(0, x_{2}\right)=-\bar{V}_{2}^{0}\left(0, x_{2}\right) \\
\bar{V}_{2}^{1}\left(\tau_{2}, 0\right)+\bar{S}^{1}\left(\tau_{2}\right)=0 \\
\frac{\partial \bar{V}_{2}^{1}}{\partial x_{2}}\left(\tau_{2}, 0\right)+\bar{S}^{1}\left(\tau_{2}\right)=\bar{V}_{2}^{0}\left(\tau_{2}, 0\right)-\frac{\partial \bar{V}_{2}^{0}}{\partial x_{2}}\left(\tau_{2}, 0\right)-1 \\
\lim _{x_{2} \rightarrow \infty} \bar{V}_{2}^{1}\left(\tau_{2}, x_{2}\right)=0
\end{array}\right.
$$


and

$$
\left\{\begin{array}{l}
\mathcal{L}_{2}\left[\bar{V}_{2}^{n}\left(\tau_{2}, x_{2}\right)\right]=\left.n \frac{\partial^{n-1} \tilde{\mathcal{A}}_{2}}{\partial p^{n-1}}\right|_{p=0} \\
\bar{V}_{2}^{n}\left(0, x_{2}\right)=0 \\
\bar{V}_{2}^{n}\left(\tau_{2}, 0\right)+\bar{S}^{n}\left(\tau_{2}\right)=0, \quad \text { if } n \geq 2 \\
\frac{\partial V_{2}^{n}}{\partial x_{2}}\left(\tau_{2}, 0\right)+\bar{S}^{n}\left(\tau_{2}\right)=0 \\
\lim _{x_{2} \rightarrow \infty} \bar{V}_{2}^{n}\left(\tau_{2}, x_{2}\right)=0
\end{array}\right.
$$

Here, $\tilde{\mathcal{A}}_{i}=\mathcal{L}_{i}\left[\bar{V}_{i}\right]-\mathcal{A}_{i}, i=1$, 2. After eliminating $\bar{S}^{n}\left(\tau_{i}\right)$ from the two boundary conditions at $x_{i}=0$ in the systems, then the systems can be written in a general form,

$$
\left\{\begin{array}{l}
\mathcal{L}_{1}\left[\bar{V}_{1}^{n}\left(\tau_{1}, x_{1}\right)\right]=f_{1}^{n}\left(\tau_{1}, x_{1}\right) \\
\bar{V}_{1}^{n}\left(0, x_{1}\right)=\psi_{1}^{n}\left(x_{1}\right) \\
\frac{\partial V_{1}^{n}}{\partial x_{1}}\left(\tau_{1}, 0\right)-\bar{V}_{1}^{n}\left(\tau_{1}, 0\right)=\phi_{1}^{n}\left(\tau_{1}\right) \\
\bar{V}_{1}^{n}\left(\tau_{1}, \infty\right)=0
\end{array}\right.
$$

where

$$
\begin{gathered}
f_{1}^{n}\left(\tau_{1}, x_{1}\right)=\left\{\begin{array}{l}
-\mathcal{L}_{1}\left[\bar{V}_{1}^{0}\left(\tau_{1}, x_{1}\right)\right]+\tilde{\mathcal{A}}_{1}\left(\tau_{1}, x_{1}, 0\right), \quad \text { if } n=1 \\
\left.n \frac{\partial^{n-1} \mathcal{A}_{1}}{\partial p^{n-1}}\right|_{p=0}, \text { if } n \geq 2
\end{array}\right. \\
\psi_{1}^{n}\left(x_{1}\right)=\left\{\begin{array}{c}
-\bar{V}_{1}^{0}\left(0, x_{1}\right), \quad \text { if } n=1 \\
0, \quad \text { if } n \geq 2
\end{array}\right. \\
\phi_{1}^{n}\left(\tau_{1}\right)=\left\{\begin{array}{l}
\bar{V}_{1}^{0}\left(\tau_{1}, 0\right)-\frac{\partial \bar{V}_{1}^{0}}{\partial x_{1}}\left(\tau_{1}, 0\right)-1, \text { if } n=1 \\
0, \quad \text { if } n \geq 2
\end{array}\right.
\end{gathered}
$$

Additionally,

$$
\left\{\begin{array}{l}
\mathcal{L}_{2}\left[\bar{V}_{2}^{n}\left(\tau_{2}, x_{2}\right)\right]=f_{2}^{n}\left(\tau_{2}, x_{2}\right) \\
\bar{V}_{2}^{n}\left(0, x_{2}\right)=\psi_{2}^{n}\left(x_{2}\right) \\
\frac{\partial V_{2}^{n}}{\partial x_{2}}\left(\tau_{2}, 0\right)-\bar{V}_{2}^{n}\left(\tau_{2}, 0\right)=\phi_{2}^{n}\left(\tau_{2}\right) \\
\bar{V}_{2}^{n}\left(\tau_{2}, \infty\right)=0
\end{array}\right.
$$

where

$$
\begin{gathered}
f_{2}^{n}\left(\tau_{2}, x_{2}\right)=\left\{\begin{array}{l}
-\mathcal{L}_{2}\left[\bar{V}_{2}^{0}\left(\tau_{2}, x_{2}\right)\right]+\tilde{\mathcal{A}}_{2}\left(\tau_{2}, x_{2}, 0\right), \quad \text { if } n=1 \\
\left.n \frac{\partial^{n-1} \mathcal{A}_{2}}{\partial p^{n-1}}\right|_{p=0}, \text { if } n \geq 2
\end{array}\right. \\
\psi_{2}^{n}\left(x_{2}\right)=\left\{\begin{array}{l}
-\bar{V}_{2}^{0}\left(0, x_{2}\right), \quad \text { if } n=1 \\
0, \quad \text { if } n \geq 2
\end{array}\right. \\
\phi_{2}^{n}\left(\tau_{2}\right)=\left\{\begin{array}{l}
\bar{V}_{2}^{0}\left(\tau_{2}, 0\right)-\frac{\partial \bar{V}_{2}^{0}}{\partial x_{2}}\left(\tau_{2}, 0\right)-1, \text { if } n=1 \\
0, \quad \text { if } n \geq 2
\end{array}\right.
\end{gathered}
$$

Now, let us apply the following transformation

$$
\bar{V}_{i}^{n}\left(\tau_{i}, x_{i}\right)=\exp \left\{-\left(\gamma_{i}-1\right) x_{i} / 2-\left(\gamma_{i}+1\right)^{2} \tau_{i} / 4\right\} \bar{U}_{i}^{n}\left(\tau_{i}, x_{i}\right),
$$


so that (45) and (49) can be expressed in the form of a standard nonhomogeneous diffusion system:

$$
\begin{aligned}
& \left\{\begin{array}{l}
\frac{\partial \bar{U}_{1}^{n}}{\partial \tau_{1}}-\frac{\partial^{2} \bar{U}_{1}^{n}}{\partial x_{1}^{2}}=\exp \left\{\left(\gamma_{1}-1\right) x_{1} / 2+\left(\gamma_{1}+1\right)^{2} \tau_{1} / 4\right\} f_{1}^{n}\left(\tau_{1}, x_{1}\right) \\
\bar{U}_{1}^{n}\left(0, x_{1}\right)=\exp \left\{\left(\gamma_{1}-1\right) x_{1} / 2\right\} \psi_{1}^{n}\left(x_{1}\right) \\
\frac{\partial \bar{U}_{1}^{n}}{\partial x_{1}}\left(\tau_{1}, 0\right)-\frac{1}{2}\left(\gamma_{1}+1\right) \bar{U}_{1}^{n}\left(\tau_{1}, 0\right)=\exp \left\{\left(\gamma_{1}+1\right)^{2} \tau_{1} / 4\right\} \phi_{1}^{n}\left(\tau_{1}\right) \\
\lim _{x_{1} \rightarrow \infty} \bar{U}_{1}^{n}\left(\tau_{1}, x_{1}\right)=0
\end{array}\right. \\
& \left\{\begin{array}{l}
\frac{\partial \bar{u}_{2}^{n}}{\partial \tau_{2}}-\frac{\partial^{2} \bar{U}_{2}^{n}}{\partial x_{2}^{2}}=\exp \left\{\left(\gamma_{2}-1\right) x_{2} / 2+\left(\gamma_{2}+1\right)^{2} \tau_{2} / 4\right\} f_{2}^{n}\left(\tau_{2}, x_{2}\right) \\
\bar{U}_{2}^{n}\left(0, x_{2}\right)=\exp \left\{\left(\gamma_{2}-1\right) x_{2} / 2\right\} \psi_{2}^{n}\left(x_{2}\right) \\
\frac{\partial \bar{U}_{2}^{n}}{\partial x_{2}}\left(\tau_{2}, 0\right)-\frac{1}{2}\left(\gamma_{2}+1\right) \bar{U}_{2}^{n}\left(\tau_{2}, 0\right)=\exp \left\{\left(\gamma_{2}+1\right)^{2} \tau_{2} / 4\right\} \phi_{2}^{n}\left(\tau_{2}\right) \\
\lim _{x_{2} \rightarrow \infty} \bar{U}_{2}^{n}\left(\tau_{2}, x_{2}\right)=0
\end{array}\right.
\end{aligned}
$$

An explicit solution of Equations (54) and (55) at each order (i.e., with each $n$ ) can now be found by splitting the linear problem into three problems, a method that is commonly used in solving linear PDEs. For further details of the solution procedure we refer to Carslaw and Jaeger (1959). The final explicit solution at each order is then:

$$
\begin{aligned}
\bar{u}_{1}^{n}\left(\tau_{1}, x_{1}\right) & =\int_{0}^{\infty} \exp \left\{\left(\gamma_{1}-1\right) \xi_{1} / 2\right\} \psi_{1}^{n}\left(\xi_{1}\right) G\left(x_{1}, \xi_{1}, \tau_{1}\right) d \xi_{1} \\
& -\int_{0}^{\tau_{1}} \exp \left\{\left(\gamma_{1}+1\right)^{2} \tau_{1} / 4\right\} \phi_{1}^{n}\left(\eta_{1}\right) G\left(x_{1}, 0, \tau_{1}-\eta_{1}\right) d \eta_{1} \\
& =\int_{0}^{\tau_{1}} \int_{0}^{\infty} \exp \left\{\left(\gamma_{1}-1\right) x_{1} / 2+\left(\gamma_{1}+1\right)^{2} \tau_{1} / 4\right\} f_{1}^{n}\left(\eta_{1}, \xi_{1}\right) G\left(x_{1}, \xi_{1}, \tau_{1}-\eta_{1}\right) d \xi_{1} d \eta_{1},
\end{aligned}
$$

where

$$
\begin{aligned}
G\left(x_{1}, \xi_{1}, \tau_{1}\right) & =\frac{1}{2 \sqrt{\pi \tau_{1}}}\left\{\exp \left[\frac{-\left(x_{1}-\xi_{1}\right)^{2}}{4 \tau_{1}}\right]+\exp \left[\frac{-\left(x_{1}+\xi_{1}\right)^{2}}{4 \tau_{1}}\right]\right. \\
& -\left(\gamma_{1}+1\right) \sqrt{\pi \tau_{1}} \exp \left[\frac{\left(\gamma_{1}+1\right)^{2}}{4}+\frac{\left(\gamma_{1}+1\right)\left(x_{1}+\xi_{1}\right)}{2}\right. \\
& \left.\left.\times \operatorname{erfc}\left(\frac{x_{1}+\xi_{1}}{2 \sqrt{\tau_{1}}}+\frac{1}{2}\left(\gamma_{1}+1\right) \sqrt{\tau_{1}}\right)\right]\right\}
\end{aligned}
$$

Additionally,

$$
\begin{aligned}
\bar{U}_{2}^{n}\left(\tau_{2}, x_{2}\right) & =\int_{0}^{\infty} \exp \left\{\left(\gamma_{2}-1\right) \xi_{2} / 2\right\} \psi_{2}^{n}\left(\xi_{2}\right) G\left(x_{2}, \xi_{2}, \tau_{2}\right) d \xi_{2} \\
& -\int_{0}^{\tau_{2}} \exp \left\{\left(\gamma_{2}+1\right)^{2} \tau_{2} / 4\right\} \phi_{2}^{n}\left(\eta_{2}\right) G\left(x_{2}, 0, \tau_{2}-\eta_{2}\right) d \eta_{2} \\
& =\int_{0}^{\tau_{2}} \int_{0}^{\infty} \exp \left\{\left(\gamma_{2}-1\right) x_{2} / 2+\left(\gamma_{2}+1\right)^{2} \tau_{2} / 4\right\} f_{2}^{n}\left(\eta_{2}, \xi_{2}\right) G\left(x_{2}, \xi_{2}, \tau_{2}-\eta_{2}\right) d \xi_{2} d \eta_{2},
\end{aligned}
$$

where

$$
\begin{aligned}
G\left(x_{2}, \xi_{2}, \tau_{2}\right) & =\frac{1}{2 \sqrt{\pi \tau_{2}}}\left\{\exp \left[\frac{-\left(x_{2}-\xi_{2}\right)^{2}}{4 \tau_{2}}\right]+\exp \left[\frac{-\left(x_{2}+\xi_{2}\right)^{2}}{4 \tau_{2}}\right]\right. \\
& -\left(\gamma_{2}+1\right) \sqrt{\pi \tau_{2}} \exp \left[\frac{\left(\gamma_{2}+1\right)^{2}}{4}+\frac{\left(\gamma_{2}+1\right)\left(x_{2}+\xi_{2}\right)}{2}\right. \\
& \left.\left.\times \operatorname{erfc}\left(\frac{x_{2}+\xi_{2}}{2 \sqrt{\tau_{2}}}+\frac{1}{2}\left(\gamma_{2}+1\right) \sqrt{\tau_{2}}\right)\right]\right\}
\end{aligned}
$$


and $\operatorname{erfc}(x)$ denotes the complementary error function.

For the choice of an initial guess $V_{i}^{0}\left(\tau_{i}, x_{i}\right), i=1,2$, any continuous functions satisfying the limiting condition can be used, we select the corresponding European option value as the initial guess with three apparent advantages given as discussed in Zhu (2006):

1. the boundary condition as $x_{i} \rightarrow \infty$ is automatically satisfied;

2. $\mathcal{L}_{i}\left[\bar{V}_{i}^{0}\left(\tau_{i}, x_{i}\right)\right]$ will become 0 ; which we expect will foster a faster convergence of the series and it also help to simply $f_{i}^{1}\left(\tau_{i}, x_{i}\right)$ as the first term of $f_{i}^{1}\left(\tau_{i}, x_{i}\right)$ will vanish; and,

3. also, as $\bar{V}_{i}^{0}$ satisfies the condition, $\bar{V}_{i}^{0}\left(0, x_{i}\right)=0$ so $\psi_{i}^{1}\left(x_{i}\right)=0$ and as $\psi_{i}^{n}\left(x_{i}\right)=0$ for all $n \in \mathbb{N} \backslash\{1\}$, the integral containing $\psi_{i}^{n}\left(x_{i}\right)$ is eliminated.

Zhu et al. (2012) provides the analytic solution of a European put option with a two-state regime switching:

$$
\begin{aligned}
& V_{i}^{0}(t, S) \\
= & K e^{-r_{i}(T-t)}+\frac{1}{4 \pi \sqrt{2}} \sqrt{S K} e^{-\frac{1}{2}\left(r_{i}+a_{21}+a_{12}+\frac{\sigma_{1}^{2}+\sigma_{2}^{2}}{8}\right)(T-t)} \int_{0}^{\infty} \frac{(-1)^{i-1} 2 \hat{f}_{1}(\rho)\left(a_{21}+a_{12}\right)}{M(\rho)\left(\rho^{4}+\frac{1}{16}\right)\left(\sigma_{1}^{2}-\sigma_{2}^{2}\right)} \\
\times & \left\{e^{X_{i}(\rho)}\left[\left(2 \rho^{2}-\frac{1}{2}\right) \sin \left(\hat{f}_{2}(\rho)+\theta(\rho)-Y_{i}(\rho)\right)-\left(2 \rho^{2}+\frac{1}{2}\right) \cos \left(\hat{f}_{2}(\rho)+\theta(\rho)-Y_{i}(\rho)\right)\right]\right. \\
- & \left.e^{-X_{i}(\rho)}\left[\left(2 \rho^{2}-\frac{1}{2}\right) \sin \left(\hat{f}_{2}(\rho)+\theta(\rho)+Y_{i}(\rho)\right)-\left(2 \rho^{2}+\frac{1}{2}\right) \cos \left(\hat{f}_{2}(\rho)+\theta(\rho)+Y_{i}(\rho)\right)\right]\right\} \\
+ & \frac{2 \hat{f}_{1}(\rho)}{M(\rho)}\left\{e^{X_{i}(\rho)}\left[\sin \left(\hat{f}_{2}(\rho)+\theta(\rho)-Y_{i}(\rho)\right)+\cos \left(\hat{f}_{2}(\rho)+\theta(\rho)-Y_{i}(\rho)\right)\right]\right. \\
- & \left.e^{-X_{i}(\rho)}\left[\sin \left(\hat{f}_{2}(\rho)+\theta(\rho)+Y_{i}(\rho)\right)+\cos \left(\hat{f}_{2}(\rho)+\theta(\rho)+Y_{i}(\rho)\right)\right]\right\} \\
+ & \frac{\hat{f}_{1}(\rho)}{\rho^{4}+\frac{1}{16}}\left\{e^{X_{i}(\rho)}\left[\left(2 \rho^{2}-\frac{1}{2}\right) \sin \left(\hat{f}_{2}(\rho)-Y_{i}(\rho)\right)-\left(2 \rho^{2}+\frac{1}{2}\right) \cos \left(\hat{f}_{2}(\rho)-Y_{i}(\rho)\right)\right]\right. \\
+ & \left.e^{-X_{i}(\rho)}\left[\left(2 \rho^{2}-\frac{1}{2}\right) \sin \left(\hat{f}_{2}(\rho)+Y_{i}(\rho)\right)-\left(2 \rho^{2}+\frac{1}{2}\right) \cos \left(\hat{f}_{2}(\rho)+Y_{i}(\rho)\right)\right]\right\} d \rho,
\end{aligned}
$$$$
\text { for } i=1,2 \text {, where }
$$

$$
\begin{gathered}
\tau=\frac{\sigma_{1}^{2}-\sigma_{2}^{2}}{4}(T-t), \quad \alpha=\frac{2\left(a_{12}-a_{21}\right)}{\sigma_{1}^{2}-\sigma_{2}^{2}}, \quad \mu^{2}=\frac{4 a_{12} a_{21}}{\left(\sigma_{1}^{2}-\sigma_{2}^{2}\right)^{2}}, \\
M(\rho)=\left\{\left[\left(\frac{1}{4}+\alpha\right)^{2}-\rho^{4}+\mu^{2}\right]^{2}+4 \rho^{4}\left(\frac{1}{4}+\alpha\right)^{2}\right\}^{\frac{1}{4}}, \\
\theta(\rho)=\frac{1}{2} \tan ^{-1}\left[\frac{2 \rho^{2}\left(\frac{1}{4}+\alpha\right)}{\left(\frac{1}{4}+\alpha\right)^{2}-\rho^{4}+\mu^{2}}\right], \\
X_{i}(\rho)=(-1)^{i-1} M(\rho) \tau \cos \theta(\rho), \quad Y_{i}(\rho)=(-1)^{i-1} M(\rho) \tau \sin \theta(\rho)
\end{gathered}
$$

and

$$
\hat{f}_{1}(\rho)=e^{-\frac{\rho}{\sqrt{2}}\left|\ln \left(\frac{S}{R}\right)+r_{1}(T-t)\right|}, \quad \hat{f}_{2}(\rho)=\frac{\rho^{2}}{4}\left(\sigma_{1}^{2}+\sigma_{2}^{2}\right)(T-t)-\frac{\rho}{\sqrt{2}}\left|\ln \left(\frac{S}{K}\right)+r_{1}(T-t)\right| .
$$

The vanishing of $\psi_{i}^{n}\left(x_{i}\right)$ allows us to write the solution $\bar{U}_{i}^{n}\left(\tau_{i}, x_{i}\right)$ in a simple and closed form: 


$$
\begin{aligned}
& \bar{U}_{1}^{n}\left(\tau_{1}, x_{1}\right)=-\frac{2}{\sqrt{\pi}} e^{\left(\gamma_{1}+1\right)^{2} \tau_{1} / 4} \int_{0}^{\infty} e^{-\left(\gamma_{1}+1\right) \eta_{1} / 2} \int_{\left(x_{1}+\eta_{1}\right) / 2 \sqrt{\tau_{1}}}^{\infty} \phi_{1}^{n}\left(\tau_{1}-\frac{\left(x_{1}+\eta_{1}\right)^{2}}{4 \xi_{1}^{2}}\right) \\
& \times e^{-\left[\left(\gamma_{1}+1\right)\left(x_{1}+\eta_{1}\right) / 4 \xi_{1}\right]^{2}-\xi_{1}^{2}} d \xi_{1} d \eta_{1}+\int_{0}^{\tau_{1}}\left\{\frac { e ^ { ( \gamma _ { 1 } + 1 ) ^ { 2 } \eta _ { 1 } / 4 } } { \sqrt { \pi } } \left[e^{\left(\gamma_{1}-1\right) x_{1} / 2}\right.\right. \\
& \times \quad \int_{-x_{1} / 2 \sqrt{\tau_{1}-\eta_{1}}}^{x_{1} / 2 \sqrt{\tau_{1}-\eta_{1}}} f_{1}^{n}\left(\eta_{1}, 2 \sqrt{\tau_{1}-\eta_{1}} \xi_{1}+x_{1}\right) e^{\left(\gamma_{1}-1\right) \sqrt{\tau_{1}-\eta_{1}} \xi_{1}-\xi_{1}^{2}} d \xi_{1} \\
& +\int_{x_{1} / 2 \sqrt{\tau_{1}-\eta_{1}}}^{\infty}\left[e^{\left(\gamma_{1}-1\right) x_{1} / 2} f_{1}^{n}\left(\eta_{1}, 2 \sqrt{\tau_{1}-\eta_{1}} \xi_{1}+x_{1}\right)\right. \\
& \left.\left.+e^{-\left(\gamma_{1}-1\right) x_{1} / 2} f_{1}^{n}\left(\eta_{1}, 2 \sqrt{\tau_{1}-\eta_{1}} \xi_{1}-x_{1}\right)\right] \times e^{\left(\gamma_{1}-1\right) \sqrt{\tau_{1}-\eta_{1}} \xi_{1}-\xi_{1}^{2}} d \xi_{1}\right] \\
& -\left(\gamma_{1}+1\right) \sqrt{\tau_{1}-\eta_{1}} e^{-\left(\gamma_{1}-1\right) x_{1} / 2+\left(\gamma_{1}+1\right)^{2} \tau_{1} / 4} \int_{x_{1} / 2 \sqrt{\tau_{1}-\eta_{1}}}^{\infty} f_{1}^{n}\left(\eta_{1}, 2 \sqrt{\tau_{1}-\eta_{1}} \xi_{1}-x_{1}\right) \\
& \left.\times \quad e^{2 \gamma_{1} \sqrt{\tau_{1}-\eta_{1}} \xi_{1}} \operatorname{erfc}\left(\xi_{1}+\frac{\left(\gamma_{1}+1\right)}{2} \sqrt{\tau_{1}-\eta_{1}}\right) d \xi_{1}\right\} d \eta_{1}
\end{aligned}
$$

$$
\begin{aligned}
& \bar{U}_{2}^{n}\left(\tau_{2}, x_{2}\right)=-\frac{2}{\sqrt{\pi}} e^{\left(\gamma_{2}+1\right)^{2} \tau_{2} / 4} \int_{0}^{\infty} e^{-\left(\gamma_{2}+1\right) \eta_{2} / 2} \int_{\left(x_{2}+\eta_{2}\right) / 2 \sqrt{\tau_{2}}}^{\infty} \phi_{2}^{n}\left(\tau_{2}-\frac{\left(x_{2}+\eta_{2}\right)^{2}}{4 \xi_{2}^{2}}\right) \\
& \times e^{-\left[\left(\gamma_{2}+1\right)\left(x_{2}+\eta_{2}\right) / 4 \xi_{2}\right]^{2}-\xi_{2}^{2}} d \xi_{2} d \eta_{2}+\int_{0}^{\tau_{2}}\left\{\frac { e ^ { ( \gamma _ { 2 } + 1 ) ^ { 2 } \eta _ { 2 } / 4 } } { \sqrt { \pi } } \left[e^{\left(\gamma_{2}-1\right) x_{2} / 2}\right.\right. \\
& \times \int_{-x_{2} / 2 \sqrt{\tau_{2}-\eta_{2}}}^{x_{2} / 2 \sqrt{\tau_{2}-\eta_{2}}} f_{2}^{n}\left(\eta_{2}, 2 \sqrt{\tau_{2}-\eta_{2}} \xi_{2}+x_{2}\right) e^{\left(\gamma_{2}-1\right) \sqrt{\tau_{2}-\eta_{2}} \xi_{2}-\xi_{2}^{2}} d \xi_{2} \\
& +\int_{x_{2} / 2 \sqrt{\tau_{2}-\eta_{2}}}^{\infty}\left[e^{\left(\gamma_{2}-1\right) x_{2} / 2} f_{2}^{n}\left(\eta_{2}, 2 \sqrt{\tau_{2}-\eta_{2}} \xi_{2}+x_{2}\right)\right. \\
& \left.\left.+e^{-\left(\gamma_{2}-1\right) x_{2} / 2} f_{2}^{n}\left(\eta_{2}, 2 \sqrt{\tau_{2}-\eta_{2}} \xi_{2}-x_{2}\right)\right] \times e^{\left(\gamma_{2}-1\right) \sqrt{\tau_{2}-\eta_{2}} \xi_{2}-\xi_{2}^{2}} d \xi_{2}\right] \\
& -\left(\gamma_{2}+1\right) \sqrt{\tau_{2}-\eta_{2}} e^{-\left(\gamma_{2}-1\right) x_{2} / 2+\left(\gamma_{2}+1\right)^{2} \tau_{2} / 4} \int_{x_{2} / 2 \sqrt{\tau_{2}-\eta_{2}}}^{\infty} f_{2}^{n}\left(\eta_{2}, 2 \sqrt{\tau_{2}-\eta_{2}} \xi_{2}-x_{2}\right) \\
& \left.\times \quad e^{2 \gamma_{2} \sqrt{\tau_{2}-\eta_{2}} \xi_{2}} \operatorname{erfc}\left(\xi_{2}+\frac{\left(\gamma_{2}+1\right)}{2} \sqrt{\tau_{2}-\eta_{2}}\right) d \xi_{2}\right\} d \eta_{2}
\end{aligned}
$$

Once $\bar{V}_{i}^{n}\left(\tau_{i}, x_{i}\right), i=1,2$ are found at each order by substituting Equations (61) and (62) back into Equation (53), $\bar{S}^{n}\left(\tau_{i}\right)$ can easily be found from the third equation of Equations (41)-(44), i.e.,

$$
\begin{aligned}
& \bar{S}^{n}\left(\tau_{1}\right)=-\bar{V}_{1}^{n}\left(\tau_{1}, 0\right), \\
& \bar{S}^{n}\left(\tau_{2}\right)=-\bar{V}_{2}^{n}\left(\tau_{2}, 0\right) .
\end{aligned}
$$

Upon finding the coefficients $\bar{V}_{i}^{n}\left(\tau_{i}, x_{i}\right)$ and $\bar{S}^{n}\left(\tau_{i}\right)$ from (43), (61)-(64), the final solution can be written in terms of a series of infinitely many terms, as

$$
\left\{\begin{array}{l}
V_{1}\left(\tau_{1}, x_{1}\right)=\bar{V}_{1}\left(\tau_{1}, x_{1}, 1\right)=\sum_{n=0}^{\infty} \frac{\bar{V}_{1}^{n}\left(\tau_{1}, x_{1}\right)}{n !} \\
S_{f_{1}}\left(\tau_{1}\right)=\bar{S}_{f_{1}}\left(\tau_{1}, 1\right)=\sum_{n=0}^{\infty} \frac{\bar{S}^{n}\left(\tau_{1}\right)}{n !}
\end{array}\right.
$$




$$
\left\{\begin{array}{l}
V_{2}\left(\tau_{2}, x_{2}\right)=\bar{V}_{2}\left(\tau_{2}, x_{2}, 1\right)=\sum_{n=0}^{\infty} \frac{\bar{V}_{2}^{n}\left(\tau_{2}, x_{2}\right)}{n !} \\
S_{f_{2}}\left(\tau_{2}\right)=\bar{S}_{f_{2}}\left(\tau_{2}, 1\right)=\sum_{n=0}^{\infty} \frac{\bar{S}^{n}\left(\tau_{2}\right)}{n !}
\end{array}\right.
$$

We also derive the exact, analytic, and closed-form formula for the optimal exercise boundaries $S_{f_{i}}\left(\tau_{i}\right), i=1,2$ in the form:

$$
\begin{aligned}
S_{f_{1}}\left(\tau_{1}\right) & =\frac{2}{\sqrt{\pi}} e^{-\left(\gamma_{1}-1\right) x_{1} / 2-\left(\gamma_{1}+1\right)^{2} \tau_{1} / 4} \sum_{n=0}^{\infty} \frac{1}{n !}\left\{e^{\left(\gamma_{1}+1\right)^{2} \tau_{1} / 4}\right. \\
& \times \int_{0}^{\infty} e^{-\left(\gamma_{1}+1\right) \eta_{1} / 2} \int_{\eta_{1} / 2 \sqrt{\tau_{1}}}^{\infty} \phi_{1}^{n}\left(\tau_{1}-\frac{\eta_{1}^{2}}{4 \xi_{1}^{2}}\right) \\
& \times e^{-\left[\left(\gamma_{1}+1\right) \eta_{1} / 4 \xi_{1}\right]^{2}-\xi_{1}^{2}} d \xi_{1} d \eta_{1} \\
& +\int_{0}^{\tau_{1}}\left[e^{\left(\gamma_{1}+1\right)^{2} \eta_{1} / 4} \int_{0}^{\infty} f_{1}^{n}\left(\eta_{1}, 2 \sqrt{\tau_{1}-\eta_{1}} \xi_{1}\right) e^{\left(\gamma_{1}-1\right) \sqrt{\tau_{1}-\eta_{1}} \xi_{1}-\xi_{1}^{2}} d \xi_{1}\right. \\
& -\frac{\sqrt{\pi}}{2}\left(\gamma_{1}+1\right) \sqrt{\tau_{1}-\eta_{1}} e^{\left(\gamma_{1}+1\right)^{2} \tau_{1} / 4} \int_{0}^{\infty} f_{1}^{n}\left(\eta_{1}, 2 \sqrt{\tau_{1}-\eta_{1}} \xi_{1}\right) \\
& \left.\left.\times e^{2 \gamma_{1} \sqrt{\tau_{1}-\eta_{1}} \xi_{1}} \operatorname{erfc}\left(\xi_{1}+\frac{\left(\gamma_{1}+1\right)}{2} \sqrt{\tau_{1}-\eta_{1}}\right) d \xi_{1}\right] d \eta_{1}\right\},
\end{aligned}
$$

and

$$
\begin{aligned}
S_{f_{2}}\left(\tau_{2}\right) & =\frac{2}{\sqrt{\pi}} e^{-\left(\gamma_{2}-1\right) x_{2} / 2-\left(\gamma_{2}+1\right)^{2} \tau_{2} / 4} \sum_{n=0}^{\infty} \frac{1}{n !}\left\{e^{\left(\gamma_{2}+1\right)^{2} \tau_{2} / 4}\right. \\
& \times \int_{0}^{\infty} e^{-\left(\gamma_{2}+1\right) \eta_{2} / 2} \int_{\eta_{2} / 2 \sqrt{\tau_{2}}}^{\infty} \phi_{2}^{n}\left(\tau_{2}-\frac{\eta_{2}^{2}}{4 \xi_{2}^{2}}\right) \\
& \times e^{-\left[\left(\gamma_{2}+1\right) \eta_{2} / 4 \xi_{2}\right]^{2}-\xi_{2}^{2}} d \xi_{2} d \eta_{2} \\
& +\int_{0}^{\tau_{2}}\left[e^{\left(\gamma_{2}+1\right)^{2} \eta_{2} / 4} \int_{0}^{\infty} f_{2}^{n}\left(\eta_{2}, 2 \sqrt{\tau_{2}-\eta_{2}} \xi_{2}\right) e^{\left(\gamma_{2}-1\right) \sqrt{\tau_{2}-\eta_{2}} \xi_{2}-\xi_{2}^{2}} d \xi_{2}\right. \\
& -\frac{\sqrt{\pi}}{2}\left(\gamma_{2}+1\right) \sqrt{\tau_{2}-\eta_{2}} e^{\left(\gamma_{2}+1\right)^{2} \tau_{2} / 4} \int_{0}^{\infty} f_{2}^{n}\left(\eta_{2}, 2 \sqrt{\tau_{2}-\eta_{2}} \xi_{2}\right) \\
& \left.\left.\times e^{2 \gamma_{2} \sqrt{\tau_{2}-\eta_{2}} \xi_{2}} \operatorname{erfc}\left(\xi_{2}+\frac{\left(\gamma_{2}+1\right)}{2} \sqrt{\tau_{2}-\eta_{2}}\right) d \xi_{2}\right] d \eta_{2}\right\} .
\end{aligned}
$$

\subsection{Transition Region}

Let us consider the transition region between the two stopping curves:

$$
\Gamma:\left\{(S, t): S_{f_{1}}(t) \leq S \leq S_{f_{2}}(t)\right\}
$$

In this region $V_{2}=V\left(t, T, S, e_{2}\right)=K-S$ and $V_{1}$ satisfies the Black-Scholes-Merton equation

$$
\frac{\partial V_{1}}{\partial t}+r_{1} S \frac{\partial V_{1}}{\partial S}+\frac{1}{2} \sigma_{1}^{2} S^{2} \frac{\partial^{2} V_{1}}{\partial S^{2}}-r_{1} V_{1}+a_{11} V_{1}-a_{11}(K-S)=0,
$$

with a terminal condition

$$
V_{1}(T, T, S)=V\left(T, T, S, e_{1}\right)=\max \{K-S, 0\} .
$$


Additionally, continuity on $S_{f_{1}}(t)$ gives:

$$
V_{1}(t, T, S)=K-S_{f_{1}}(t)
$$

and smoothness on $S_{f_{1}}(t)$ gives:

$$
\frac{\partial V_{1}}{\partial S}\left(t, T, S_{f_{1}}(t)\right)=-1 .
$$

To solve this system effectively, we shall make all of the variables dimensionless by introducing dimensionless variables

$$
V_{1}^{\prime}=\frac{V_{1}}{K}, \quad S^{\prime}=\frac{S}{K}, \quad \tau_{1}^{\prime}=\frac{\sigma_{1}^{2}}{2}(T-t)
$$

and also make the Laudau transformation

$$
x_{1}=\ln \left(\frac{S^{\prime}}{S_{f_{1}}\left(\tau_{1}\right)}\right) .
$$

With all of the primes dropped from now on, the dimensionless systems with fixed boundary conditions read

$$
\left\{\begin{array}{l}
\frac{\partial V_{1}}{\partial \tau_{1}}-\left(\gamma_{1}-1\right) \frac{\partial V_{1}}{\partial x_{1}}-\frac{\partial^{2} V_{1}}{\partial x_{1}^{2}}+\gamma_{1} V_{1}=\lambda_{1}\left(K-e^{x_{1}} S_{f_{1}}\left(\tau_{1}\right)-V_{1}\right)+\frac{1}{S_{f_{1}}\left(\tau_{1}\right)} \frac{d S_{f_{1}}\left(\tau_{1}\right)}{d \tau_{1}} \frac{\partial V_{1}}{\partial x_{1}} \\
V_{1}\left(\tau_{1}, 0\right)=1-S_{f_{1}}\left(\tau_{1}\right) \\
\frac{\partial V_{1}}{\partial x_{1}}\left(\tau_{1}, 0\right)=-S_{f_{1}}\left(\tau_{1}\right) \\
V_{1}\left(0, x_{1}\right)=0 \\
\lim _{x_{1} \rightarrow \infty} V_{1}\left(\tau_{1}, x_{1}\right)=0
\end{array}\right.
$$

We will use the homotopy analysis method to solve this nonlinear system of equations. We introduce an embedding parameter $p \in[0,1]$ and construct unknown functions $\bar{V}_{1}\left(\tau_{1}, x_{1}, p\right)$ and $\bar{S}_{f_{1}}\left(\tau_{1}, p\right)$. The zeroth-order deformation is given by

$$
\left\{\begin{array}{l}
(1-p) \mathcal{L}_{1}\left[\bar{V}_{1}\left(\tau_{1}, x_{1}, p\right)-\bar{V}_{1}^{0}\left(\tau_{1}, x_{1}\right)\right]=-p\left\{\hat{\mathcal{A}}_{1}\left[\bar{V}_{1}\left(\tau_{1}, x_{1}, p\right), \bar{S}_{f_{1}}\left(\tau_{1}, p\right)\right]\right\} \\
\bar{V}_{1}\left(0, x_{1}, p\right)=(1-p) \bar{V}_{1}^{0}\left(0, x_{1}\right) \\
\bar{V}_{1}\left(\tau_{1}, 0, p\right)+\bar{S}_{f_{1}}\left(\tau_{1}, p\right)=1 \\
\frac{\partial \bar{V}_{1}}{\partial x_{1}}\left(\tau_{1}, 0, p\right)+\bar{S}_{f_{1}}\left(\tau_{1}, p\right)=(1-p)\left[1+\frac{\partial \bar{V}_{1}^{0}}{\partial x_{1}}\left(\tau_{1}, 0\right)-\bar{V}_{1}^{0}\left(\tau_{1}, 0\right)\right] \\
\lim _{x_{1} \rightarrow \infty} \bar{V}_{1}\left(\tau_{1}, x_{1}, p\right)=0
\end{array}\right.
$$

Here, $\mathcal{L}_{1}$ is a differential operator that is defined as

$$
\mathcal{L}_{1}=\frac{\partial}{\partial \tau_{1}}-\left(\gamma_{1}-1\right) \frac{\partial}{\partial x_{1}}-\frac{\partial^{2}}{\partial x_{1}^{2}}+\gamma_{1},
$$

and $\hat{\mathcal{A}}_{1}$ is a functional defined as 


$$
\begin{aligned}
& \hat{\mathcal{A}}_{1}\left[\bar{V}_{1}\left(\tau_{1}, x_{1}, p\right), \bar{S}_{f_{1}}\left(\tau_{1}, p\right)\right] \\
= & \mathcal{L}_{1}\left(\bar{V}_{1}\right)-\lambda_{1}\left(\bar{V}_{1}+e^{x_{1}} \bar{S}_{f_{1}}\left(\tau_{1}, p\right)-K\right)-\frac{1}{\bar{S}_{f_{1}}\left(\tau_{1}, p\right)} \frac{d \bar{S}_{f_{1}}\left(\tau_{1}, p\right)}{d \tau_{1}} \frac{\partial \bar{V}_{1}}{\partial x_{1}}\left(\tau_{1}, x_{1}, p\right) .
\end{aligned}
$$

With $p=1$, we have

$$
\left\{\begin{array}{l}
\mathcal{L}_{1}\left(\bar{V}_{1}\right)-\lambda_{1}\left(\bar{V}_{1}+e^{x_{1}} \bar{S}_{f_{1}}\left(\tau_{1}, 1\right)-K\right)-\frac{1}{\bar{S}_{f_{1}}\left(\tau_{1}, 1\right)} \frac{d \bar{S}_{f_{1}}\left(\tau_{1}, 1\right)}{d \tau_{1}} \frac{\partial \bar{V}_{1}}{\partial x_{1}}\left(\tau_{1}, x_{1}, 1\right)=0 \\
\bar{V}_{1}\left(0, x_{1}, 1\right)=0 \\
\bar{V}_{1}\left(\tau_{1}, 0,1\right)=1-\bar{S}_{f_{1}}\left(\tau_{1}, 1\right) \\
\frac{\partial \bar{V}_{1}}{\partial x_{1}}\left(\tau_{1}, 0,1\right)=-\bar{S}_{f_{1}}\left(\tau_{1}, 1\right) \\
\lim _{x_{1} \rightarrow \infty} \bar{V}_{1}\left(\tau_{1}, x_{1}, 1\right)=0
\end{array}\right.
$$

When comparing with (71), it is easy to see that the solution we seek is nothing but

$$
\left\{\begin{array}{l}
V_{1}\left(\tau_{1}, x_{1}\right)=\bar{V}_{1}\left(\tau_{1}, x_{1}, 1\right) \\
S_{f_{1}}\left(\tau_{1}\right)=\bar{S}_{f_{1}}\left(\tau_{1}, 1\right)
\end{array}\right.
$$

If $p=0$, then we have

$$
\left\{\begin{array}{l}
\mathcal{L}_{1}\left[\bar{V}_{1}\left(\tau_{1}, x_{1}, 0\right)\right]=\mathcal{L}_{1}\left[\bar{V}_{1}^{0}\left(\tau_{1}, x_{1}\right)\right] \\
\bar{V}_{1}\left(0, x_{1}, 0\right)=\bar{V}_{1}^{0}\left(0, x_{1}\right) \\
\bar{V}_{1}\left(\tau_{1}, 0,0\right)+\bar{S}_{f_{1}}\left(\tau_{1}, 0\right)=1 \\
\frac{\partial \bar{V}_{1}}{\partial x_{1}}\left(\tau_{1}, 0,0\right)+\bar{S}_{f_{1}}\left(\tau_{1}, 0\right)=\left[1+\frac{\partial \bar{V}_{1}^{0}}{\partial x_{1}}\left(\tau_{1}, 0\right)-\bar{V}_{1}^{0}\left(\tau_{1}, 0\right)\right] \\
\lim _{x_{1} \rightarrow \infty} \bar{V}_{1}\left(\tau_{1}, x_{1}, 0\right)=0
\end{array}\right.
$$

Clearly, the solution of the differential system (77) is

$$
\left\{\begin{array}{l}
\bar{V}_{1}\left(\tau_{1}, x_{1}, 0\right)=\bar{V}_{1}^{0}\left(\tau_{1}, x_{1}\right) \\
\bar{S}_{f_{1}}\left(\tau_{1}, 0\right)=1-\bar{V}_{1}^{0}\left(\tau_{1}, 0\right)=\bar{S}^{0}\left(\tau_{1}\right)
\end{array}\right.
$$

so long as the initial guess $\bar{V}_{1}^{0}\left(\tau_{1}, x_{1}\right)$ satisfies the condition

$$
\lim _{x_{1} \rightarrow \infty} \bar{V}_{1}^{0}\left(\tau_{1}, x_{1}\right)=0 .
$$

The initial guess $\bar{V}_{1}^{0}\left(\tau_{1}, x_{1}\right)$ is given in Equation (60). To determine the values of $\bar{V}_{1}\left(\tau_{1}, x_{1}, 1\right)$ and $\bar{S}_{f_{1}}\left(\tau_{1}, 1\right)$, we can apply a Taylor's series expansion of $p$ to the functions $\bar{V}_{1}\left(\tau_{1}, x_{1}, p\right)$ and $\bar{S}_{f_{1}}\left(\tau_{1}, p\right)$

$$
\left\{\begin{array}{l}
\bar{V}_{1}\left(\tau_{1}, x_{1}, p\right)=\sum_{n=0}^{\infty} \frac{\bar{V}_{1}^{n}\left(\tau_{1}, x_{1}\right)}{n !} p^{n} \\
\bar{S}_{f_{1}}\left(\tau_{1}, p\right)=\sum_{n=0}^{\infty} \frac{\bar{S}^{n}\left(\tau_{1}\right)}{n !} p^{n}
\end{array}\right.
$$

where

$$
\left\{\begin{array}{l}
\bar{V}_{1}^{n}\left(\tau_{1}, x_{1}\right)=\left.\frac{\partial^{n}}{\partial p^{n}} \bar{V}_{1}\left(\tau_{1}, x_{1}, p\right)\right|_{p=0} \\
\bar{S}^{n}\left(\tau_{1}\right)=\left.\frac{\partial^{n}}{\partial p^{n}} \bar{S}_{f_{1}}\left(\tau_{1}, p\right)\right|_{p=0}
\end{array}\right.
$$


In order to determine the coefficients in the above Taylor series expansion, we derive a set of governing equations by differentiating the zeroth-order (72), with respect to $p$ and then setting $p=0$. After this process, we obtain

$$
\left\{\begin{array}{l}
\mathcal{L}_{1}\left[\bar{V}_{1}^{1}\left(\tau_{1}, x_{1}\right)\right]=-\mathcal{L}_{1}\left[\bar{V}_{1}^{0}\left(\tau_{1}, x_{1}\right)\right]+\overline{\mathcal{A}}_{1}\left(\tau_{1}, x_{1}, 0\right) \\
\bar{V}_{1}^{1}\left(0, x_{1}\right)=-\bar{V}_{1}^{0}\left(0, x_{1}\right) \\
\bar{V}_{1}^{1}\left(\tau_{1}, 0\right)+\bar{S}^{1}\left(\tau_{1}\right)=0 \\
\frac{\partial \bar{V}_{1}^{1}}{\partial x_{1}}\left(\tau_{1}, 0\right)+\bar{S}^{1}\left(\tau_{1}\right)=\bar{V}_{1}^{0}\left(\tau_{1}, 0\right)-\frac{\partial \bar{V}_{1}^{0}}{\partial x_{1}}\left(\tau_{1}, 0\right)-1 \\
\lim _{x_{1} \rightarrow \infty} \bar{V}_{1}^{1}\left(\tau_{1}, x_{1}\right)=0
\end{array}\right.
$$

and

$$
\left\{\begin{array}{l}
\mathcal{L}_{1}\left[\bar{V}_{1}^{n}\left(\tau_{1}, x_{1}\right)\right]=\left.n \frac{\partial^{n-1} \overline{\mathcal{A}}_{1}}{\partial p^{n-1}}\right|_{p=0} \\
\bar{V}_{1}^{n}\left(0, x_{1}\right)=0 \\
\bar{V}_{1}^{n}\left(\tau_{1}, 0\right)+\bar{S}^{n}\left(\tau_{1}\right)=0, \quad \text { if } \quad n \geq 2 \\
\frac{\partial \bar{V}_{1}^{n}}{\partial x_{1}}\left(\tau_{1}, 0\right)+\bar{S}^{n}\left(\tau_{1}\right)=0 \\
\lim _{x_{1} \rightarrow \infty} \bar{V}_{1}^{n}\left(\tau_{1}, x_{1}\right)=0
\end{array}\right.
$$

Here, $\overline{\mathcal{A}}_{1}=\mathcal{L}\left[\bar{V}_{1}\right]-\hat{\mathcal{A}}_{1}$. After eliminating $\bar{S}^{n}\left(\tau_{1}\right)$ from the two boundary conditions at $x_{1}=0$ in the systems, then the systems can be written in a general form,

$$
\left\{\begin{array}{l}
\mathcal{L}_{1}\left[\bar{V}_{1}^{n}\left(\tau_{1}, x_{1}\right)\right]=\tilde{f}_{1}^{n}\left(\tau_{1}, x_{1}\right) \\
\bar{V}_{1}^{n}\left(0, x_{1}\right)=\psi_{1}^{n}\left(x_{1}\right) \\
\frac{\partial V_{1}^{n}}{\partial x_{1}}\left(\tau_{1}, 0\right)-\bar{V}_{1}^{n}\left(\tau_{1}, 0\right)=\phi_{1}^{n}\left(\tau_{1}\right) \\
\bar{V}_{1}^{n}\left(\tau_{1}, \infty\right)=0
\end{array}\right.
$$

where

$$
\begin{gathered}
\tilde{f}_{1}^{n}\left(\tau_{1}, x_{1}\right)=\left\{\begin{array}{l}
-\mathcal{L}_{1}\left[\bar{V}_{1}^{0}\left(\tau_{1}, x_{1}\right)\right]+\overline{\mathcal{A}}_{1}\left(\tau_{1}, x_{1}, 0\right), \quad \text { if } n=1 \\
n \frac{\left.\partial^{n-1} \overline{\mathcal{A}}_{1}\right|_{p=0}, \text { if } n \geq 2}{\partial p^{n-1}} n
\end{array}\right. \\
\psi_{1}^{n}\left(x_{1}\right)=\left\{\begin{array}{c}
-\bar{V}_{1}^{0}\left(0, x_{1}\right), \quad \text { if } n=1 \\
0, \quad \text { if } n \geq 2
\end{array}\right. \\
\phi_{1}^{n}\left(\tau_{1}\right)=\left\{\begin{array}{l}
\bar{V}_{1}^{0}\left(\tau_{1}, 0\right)-\frac{\partial \bar{V}_{1}^{0}}{\partial x_{1}}\left(\tau_{1}, 0\right)-1, \text { if } n=1 \\
0, \quad \text { if } n \geq 2
\end{array}\right.
\end{gathered}
$$

Now, we apply the following transformation

$$
\bar{V}_{1}^{n}\left(\tau_{1}, x_{1}\right)=\exp \left\{-\left(\gamma_{1}-1\right) x_{1} / 2-\left(\gamma_{1}+1\right)^{2} \tau_{1} / 4\right\} \bar{U}_{1}^{n}\left(\tau_{1}, x_{1}\right),
$$

so that (84) can be written in the form of a standard nonhomogeneous diffusion system:

$$
\left\{\begin{array}{l}
\frac{\partial \bar{u}_{1}^{n}}{\partial \tau_{1}}-\frac{\partial^{2} \bar{U}_{1}^{n}}{\partial x_{1}^{2}}=\exp \left\{\left(\gamma_{1}-1\right) x_{1} / 2+\left(\gamma_{1}+1\right)^{2} \tau_{1} / 4\right\} \tilde{f}_{1}^{n}\left(\tau_{1}, x_{1}\right) \\
\bar{U}_{1}^{n}\left(0, x_{1}\right)=\exp \left\{\left(\gamma_{1}-1\right) x_{1} / 2\right\} \psi_{1}^{n}\left(x_{1}\right) \\
\frac{\partial u_{1}^{n}}{\partial x_{1}}\left(\tau_{1}, 0\right)-\frac{1}{2}\left(\gamma_{1}+1\right) \bar{U}_{1}^{n}\left(\tau_{1}, 0\right)=\exp \left\{\left(\gamma_{1}+1\right)^{2} \tau_{1} / 4\right\} \phi_{1}^{n}\left(\tau_{1}\right) \\
\lim _{x_{1} \rightarrow \infty} \bar{U}_{1}^{n}\left(\tau_{1}, x_{1}\right)=0
\end{array}\right.
$$

The final explicit solution at each order is then: 


$$
\begin{aligned}
\bar{U}_{1}^{n}\left(\tau_{1}, x_{1}\right) & =\int_{0}^{\infty} \exp \left\{\left(\gamma_{1}-1\right) \xi_{1} / 2\right\} \psi_{1}^{n}\left(\xi_{1}\right) G\left(x_{1}, \xi_{1}, \tau_{1}\right) d \xi_{1} \\
& -\int_{0}^{\tau_{1}} \exp \left\{\left(\gamma_{1}+1\right)^{2} \tau_{1} / 4\right\} \phi_{1}^{n}\left(\eta_{1}\right) G\left(x_{1}, 0, \tau_{1}-\eta_{1}\right) d \eta_{1} \\
& =\int_{0}^{\tau_{1}} \int_{0}^{\infty} \exp \left\{\left(\gamma_{1}-1\right) x_{1} / 2+\left(\gamma_{1}+1\right)^{2} \tau_{1} / 4\right\} \tilde{f}_{1}^{n}\left(\eta_{1}, \xi_{1}\right) G\left(x_{1}, \xi_{1}, \tau_{1}-\eta_{1}\right) d \xi_{1} d \eta_{1},
\end{aligned}
$$

where

$$
\begin{aligned}
G\left(x_{1}, \xi_{1}, \tau_{1}\right) & =\frac{1}{2 \sqrt{\pi \tau_{1}}}\left\{\exp \left[\frac{-\left(x_{1}-\xi_{1}\right)^{2}}{4 \tau_{1}}\right]+\exp \left[\frac{-\left(x_{1}+\xi_{1}\right)^{2}}{4 \tau_{1}}\right]\right. \\
& -\left(\gamma_{1}+1\right) \sqrt{\pi \tau_{1}} \exp \left[\frac{\left(\gamma_{1}+1\right)^{2}}{4}+\frac{\left(\gamma_{1}+1\right)\left(x_{1}+\xi_{1}\right)}{2}\right. \\
& \left.\left.\times \operatorname{erfc}\left(\frac{x_{1}+\xi_{1}}{2 \sqrt{\tau_{1}}}+\frac{1}{2}\left(\gamma_{1}+1\right) \sqrt{\tau_{1}}\right)\right]\right\} .
\end{aligned}
$$

Once $\bar{V}_{1}^{n}\left(\tau_{1}, x_{1}\right)$, are found at each order by substituting Equations (90) back into Equation (88), $\bar{S}^{n}\left(\tau_{1}\right)$ can easily be found from the third equation of Equations (82) and (83), i.e.,

$$
\bar{S}^{n}\left(\tau_{1}\right)=-\bar{V}_{1}^{n}\left(\tau_{1}, 0\right)
$$

Upon finding the coefficients $\bar{V}_{1}^{n}\left(\tau_{1}, x_{1}\right)$ and $\bar{S}^{n}\left(\tau_{1}\right)$ from (88), (90), and (92), the final solution can be written in terms of a series of infinitely many terms as

$$
\left\{\begin{array}{l}
V_{1}\left(\tau_{1}, x_{1}\right)=\bar{V}_{1}\left(\tau_{1}, x_{1}, 1\right)=\sum_{n=0}^{\infty} \frac{\bar{V}_{1}^{n}\left(\tau_{1}, x_{1}\right)}{n !}, \\
S_{f_{1}}\left(\tau_{1}\right)=\bar{S}_{f_{1}}\left(\tau_{1}, 1\right)=\sum_{n=0}^{\infty} \frac{\bar{S}^{n}\left(\tau_{1}\right)}{n !} .
\end{array}\right.
$$

\section{Conclusions}

By means of the homotopy analysis method, an exact analytical pricing formula for the American option under a two-state regime-switching economy is derived. It is shown that the optimal exercise boundary, which is the key difficulty in the valuation of American options, can be explicitly expressed in the form of infinite series. In addition, we provide a flow chart to show when a particular formula should be used for the easiness of using our solutions to price American put options under a two-state regime switching model.

Author Contributions: Conceptualization, L.C. and S.-P.Z.; methodology, S.-P.Z. and L.C.; Analysis, L.C.; Writing-original draft preparation, L.C.; Discussion, L.C. and S.-P.Z. Both authors have read and agreed to the published version of the manuscript.

Funding: This research received no external funding.

Institutional Review Board Statement: Not applicable.

Informed Consent Statement: Not applicable.

Data Availability Statement: Not applicable.

Acknowledgments: Not applicable.

Conflicts of Interest: The authors declare no conflict of interest. 


\section{References}

Alobaidi, Ghada, and Roland Mallier. 2001. Asymptotic analysis of American call options. International Journal on Mathematics and Mathematical Sciences 27: 177-88. [CrossRef]

Barone-Adesi, Giovanni, and Robert E. Whaley. 1987. Efficient analytic approximation of American option values. Journal of Finance 42: 301-20. [CrossRef]

Barone-Adesi, Giovanni, and Robert J. Elliott. 1991. Approximations for the values of American options. Stochastic Analysis and Applications 9: 115-31. [CrossRef]

Black, Fischer, and Myron Scholes. 1973. The pricing of options and corporate liabilities. Journal of Political Economy 81: 637-59. [CrossRef]

Brennan, Michael, and Eduardo Schwartz. 1977. The valuation of American put options. Journal of Finance 32: 449-62. [CrossRef]

Buffington, John, and Robert J. Elliott. 2002a. Regime switching and European options. In Stochastic Theory and Control. Edited by Lawrence Kansas. Berlin and Heidelberg: Springer, pp. 73-81.

Buffington, John, and Robert J. Elliott. 2002b. American options with regime switching. International Journal of Theoretical and Applied Finance 5: 497-514. [CrossRef]

Bunch, David S., and Hensher Johnson. 2000. The American put option and its critical stock price. The Journal of Finance 5: 2333-56. [CrossRef]

Carr, Peter. 1998. Randomization and the American put. Review of Financial Studies 11: 597-626. [CrossRef]

Carr, Peter, and Dmitri Faguet. 1996. Valuing Finite-Lived Options as Perpetual. Working paper. New York: Morgan Stanley.

Carr, Peter, Robert Jarrow, and Ravi Myneni. 1992. Alternative characterizations of American Puts. Mathematical Finance 2: 87-106. [CrossRef]

Carslaw, Horatio S., and John C. Jaeger. 1959. Conduction of Heat in Solids. Oxford: Clarendon Press.

Chan, Leunglung, and Song-Ping Zhu. 2015. An analytic formula for pricing American-style convertible bonds in a regime switching model. IMA Journal of Management Mathematics 26: 403-28. [CrossRef]

Chevalier, Etienne. 2005. Critical price near maturity for an American option on dividend-paying stock in a local volatility model. Mathematical Finance 15: 439-63. [CrossRef]

Egami, Masahiko, and Rusudan Kevkhishvili. 2020. A direct solution method for pricing options in regime-switching models. Mathematical Finance 30: 547-76. [CrossRef]

Elliott, Robert J., Lakhdar Aggoun, and John B. Moore. 1994. Hidden Markov Models: Estimation and Control. Berlin, Heidelberg and New York: Springer.

Elliott, Robert J., and John van der Hoek. 1997. An application of hidden Markov models to asset allocation problems. Finance and Stochastics 3: 229-38. [CrossRef]

Elliott, Robert J., and P. Ekkehard Kopp. 1999. Mathematics of Financial Markets. Berlin, Heidelberg and New York: Springer.

Elliott, Robert J., William C. Hunter, and Barbara M. Jamieson. 2001. Financial signal processing. International Journal of Theoretical and Applied Finance 4: 567-84. [CrossRef]

Elliott, Robert J., William P. Malcolm, and Allanus H. Tsoi. 2003. Robust parameter estimation for asset price models with Markov modulated volatilities. Journal of Economics Dynamics and Control 27: 1391-409. [CrossRef]

Elliott, Robert J., Leunglung Chan, and Tak Kuen Siu. 2005. Option Pricing and Esscher Transform Under Regime Switching. Annals of Finance 1: 423-32. [CrossRef]

Elliott, Robert J., and Andrew J. Royal. 2007. Asset prices with regime switching variance Gamma dynamics. In Hand Book on Mathematical Finance. Edited by Alain Bensoussan and Qiang Zhang. Amsterdam: Elsevier, pp. 685-711.

Evans, Jonathan, Joseph Keller, and Rachel Kuske. 2002. American options on assets with dividends near expiry. Mathematical Finance 12: 219-37. [CrossRef]

Geske, Robert, and Herb E. Johnson. 1984. The American put option valued analytically. Journal of Finance 39: 1511-1524. [CrossRef]

Geske, Robert, and Kuldeep Shastri. 1985. Valuation by approximation: A comparison of alternative option valuation techniques. Journal of Financial and Quantitative Analysis 20: 45-71. [CrossRef]

Gounden, Shashen, and John G. O'Hara. 2010. An analytic formula for the price of an American-style Asian option of floating strike type. Applied Mathematics and Computation 217: 2923-36. [CrossRef]

Guo, Xin. 2001a. Information and option pricings. Quantitative Finance 1: 38-44. [CrossRef]

Guo, Xin. 2001b. An explicit solution to an optimal stopping problem with regime switching. Journal of Applied Probability 38: 464-81. [CrossRef]

Guo, Xin, and Larry Shepp. 2001. Some optimal stopping problems with non-trivial boundaries for pricing exotic options. Journal of Applied Probability 38: 647-58. [CrossRef]

Guo, Xin, and Qing Zhang. 2004. Closed-form solutions for perpetual American put options with regime switching. SIAM Journal on Applied Mathematics 64: 2034-49.

He, Xinjiang, and Song-Ping Zhu. 2018. On full calibration of hybrid local volatility and regime-switching models. Journal of Futures Markets 38: 586-606. [CrossRef]

He, Xinjiang, and Song-Ping Zhu. 2021. A new algorithm for calibrating local regime-switching models. IMA Journal of Management Mathematics 32: 237-255. [CrossRef] 
Hon, Yiu-Chung, and Xian-Zhong Mao. 1997. A radial basis function method for solving options pricing model. Journal of Financial Engineering 8: 31-49.

Huang, Jing Zhi, Marti G. Subrahmanyam, and G. George Yu. 1996. Pricing and hedging American options: A recursive integration method. Review of Financial Studies 9: 277-300. [CrossRef]

Huang, Yiqing, Peter A. Forsyth, and George Labahn. 2011. Methods for pricing American options under regime switching. SIAM Journal on Scientific Computing 33: 2144-68. [CrossRef]

Jacka, Saul D. 1991. Optimal stopping and the American put. Mathematical Finance 1: 1-14. [CrossRef]

Jobert, Arnaud, and Leonard Chris G. Rogers. 2006. Option pricing with Markov-modulated dynamics. SIAM Journal on Control and Optimization 44: 2063-78. [CrossRef]

Johnson, Herb E. 1983. An analytical approximation for the American put price. Journal of Financial and Quantitative Analysis 18: 141-148. [CrossRef]

Ju, Nengjiu. 1998. Pricing an American option by approximating its early exercise boundary as a multipiece exponential function. Review of Financial Studies 11: 627-46. [CrossRef]

Kim, In Joon. 1990. The analytical valuation of American options. Review of Financial Studies 3: 547-72. [CrossRef]

Leung, Kwai Sun. 2013. An analytic pricing formula for lookback options under stochastic volatility. Applied Mathematics Letters 26: 145-49. [CrossRef]

Liao, Shijun. 1997. Numerically solving non-linear problems by the homotopy analysis method. Computational Mechanics 20: 530-40. [CrossRef]

Liao, Shijun, and Antonio Campo. 2002. Analytic solutions of the temperature distribution in Blasius viscous flow problems. Journal of Fluid Mechanics 453: 411-25. [CrossRef]

Liao, Shijun, and Ji-Mao Zhu. 1996. A short note on higher-order streamfunction-vorticity formulations of 2D steady state Navier-Stokes equations. International Journal for Numerical Methods in Fluids 22: 1-9.

Liao, Shijun, and Song-Ping Zhu. 1999. Solving the Liouville equation with the general boundary element method approach. Boundary Element Technology 13: 407-16.

Lu, Xiaoping, and Endah Putri. 2020. A semi-analytical valuation of American options under a two-state regime-switching economy. Physica A 538: 122968. [CrossRef]

MacMillan, L.W. 1986. An analytical approximation for the American put prices. Advances in Futures and Options Research 1: 119-39.

Mallier, Roland. 2002. Evaluating approximations to the optimal exercise boundary. Journal of Applied Mathematics 2: 71-92. [CrossRef]

McKean, Henry P. 1965. A free boundary problem for the heating function arising from a problem in mathematical economics. Industrial Management Review 6: 32-39.

Merton, Robert. 1973. Theory of rational option pricing. Bell Journal of Economics and Management Science 4: 141-83. [CrossRef]

Omberg, Edward. 1987. The valuation of American puts with exponential exercise policies. Advances in Futures and Options Research 2: $117-42$.

Ortega, James M., and Werner C. Rheinboldt. 1970. Iterative Solution of Nonlinear Equations in Several Variables. New York: Academic Press.

Yi, Fahuai. 2008. American put option with regime-switching volatility (finite time horizon)-Variational inequality approach. Mathematical Methods in Applied Sciences 31: 1461-77. [CrossRef]

Zhu, Song-Ping. 2006. An exact and explicit solution for the valuation of American put options. Quantitative Finance 6: 229-42. [CrossRef]

Zhu, Song-Ping, Alexander Badran, and Xiaoping Lu. 2012. A new exact solution for pricing European options in a two-state regime-switching economy. Computers and Mathematics with Applications 64: 2744-55. [CrossRef] 